\title{
ON THE DIMENSION OF TRIANGULAR SELF-AFFINE SETS
}

\author{
BALÁZS BÁRÁNY, MICHAŁ RAMS, AND KÁROLY SIMON
}

\begin{abstract}
As a continuation of a recent work [6] of the same authors, in this note we study the dimension theory of diagonally homogeneous triangular planar self-affine IFS.
\end{abstract}

\section{INTRODUCTION}

1.1. The theme of the paper. The dimension theory of self-affine measures and sets is so complicated that even on $\mathbb{R}^{2}$, in the diagonal case (when all the linear part of the mappings from the IFS are diagonal matrices), it is not fully understood. The authors of this note have recently investigated this question [6]. Namely, consider the diagonal self-affine IFS on the plane

$$
\mathcal{S}^{\text {diag }}:=\left\{S_{i}^{\operatorname{diag}}(x, y):=D_{i} \cdot\left(\begin{array}{l}
x \\
y
\end{array}\right)+\left(\begin{array}{c}
u_{i} \\
v_{i}
\end{array}\right)\right\}_{i=1}^{N} \text {, where } D_{i}:=\left(\begin{array}{cc}
c_{i} & 0 \\
0 & b_{i}
\end{array}\right) .
$$

The projection of the coordinate axis, naturally generates a self-similar IFS on both coordinate axis. Assume that not both of the similarity dimensions of these projected self-similar IFS are greater than one. In this case, the dimension theory of diagonal selfaffine systems on $\mathbb{R}^{2}$ are settled in [6], at least for all but a very small set of parameters.

In this note we make a step forward and consider triangular self-affine IFS. That is we assume that the linear part of the mappings from the IFS are triangular matrices (all of them are lower triangular say). More precisely, let

$$
\mathcal{S}:=\left\{S_{i}(x, y):=T_{i} \cdot\left(\begin{array}{l}
x \\
y
\end{array}\right)+\left(\begin{array}{c}
u_{i} \\
v_{i}
\end{array}\right)\right\}_{i=1}^{N}, \text { where } T_{i}:=\left(\begin{array}{cc}
c_{i} & 0 \\
d_{i} & b_{i}
\end{array}\right) .
$$

We say that $\mathcal{S}$ is diagonally homogeneous if there exists constants $b, c \in(0,1)$ such that for all $i c_{i}=c$ and $b_{i}=b$. We mostly investigate the diagonally homogeneous case, see Section 4 and Section 5 . However, in the general case, we have result in the case when affinity dimension is smaller than one, see Section 3.

1.2. History. A self-affine Iterated Function System (IFS) is a finite list of contracting affine mappings on $\mathbb{R}^{d}$. If we choose a closed ball $B \subset \mathbb{R}^{d}$ centered at the origin with sufficiently high radius then this ball will be mapped into itself by all the mappings from the IFS. The ellipses obtained by applying the mappings of the IFS, in any particular order $n$-times, on this ball $B$, are the $n$-cylinders. As $n$ tends to infinity, the shapes of many of the $n$-cylinders become more and more relatively thinner and longer. This makes

Date: August 11, 2017.

2010 Mathematics Subject Classification. Primary 28A80 Secondary 28A78.

Key words and phrases. Self-affine measures, self-affine sets, Hausdorff dimension.

The research of Bárány and Simon was partially supported by the grant OTKA K104745. Bárány was partially supported by the János Bolyai Research Scholarship of the Hungarian Academy of Sciences and ERC grant 306494. Michał Rams was supported by National Science Centre grant 2014/13/B/ST1/01033 (Poland). This work was partially supported by the grant 346300 for IMPAN from the Simons Foundation and the matching 2015-2019 Polish MNiSW fund. Part of Simon's research was supported by ICERM by supporting his participation on one of their a semester programs in 2016 . 
it possible that even if the $n$-cylinders are pairwise disjoint, in some cases, they are not effective covers of the attractor (which is the set that remains after infinite number of iterations of the mappings from the IFS on this ball $B$ above).

In 1988 Falconer introduced the notion of affinity dimension [10] for self-affine fractals. We obtain it if we replace the "most economic cover" in the definition of Hausdorff dimension with the most natural cover associated with the $n$-cylinders. In some sense the affinity dimension is the most natural guess for the Hausdorff dimension of a self-affine attractor. Since the affinity dimension $\operatorname{dim}_{\text {aff }}(\mathcal{F})$ of a self-affine IFS

$$
\mathcal{F}:=\left\{f_{i}(x)=A_{i} \cdot \mathbf{x}+\mathbf{t}_{i}\right\}_{i=1}^{N}
$$

depends only on the linear parts, therefore it remains the same if we vary the translation vectors in $\mathcal{F}$. In 1988 Falconer proved that for almost all translates of a self-affine IFS, the Hausdorff dimension of the attractor and affinity dimension of the IFS are equal, if all of the mappings from the IFS has strong enough contraction. This upper bound on the contractions was originally 1/3, which was improved 10 year later by Solomyak [26] to 1/2. Solomyak [26] also showed that the bound $1 / 2$ is optimal. For a survey of results before 2014 see e.g. [23]. In the last two years there have been a very intensive development on this field, partially due to the use of Furstenberg-Kifer measure (usually referred as Furstenberg measure). See [1, [3], [5], 4], 20], [7, [14].

1.3. Affinity dimension in the triangular case on $\mathbb{R}^{2}$. In the special case of the triangular self-affine IFS, Falconer and Miao [8, Corollary 2.6] showed that if all the matrices are (e.g. lower) triangular then the affinity dimension can be given explicitly by a formula which depends only on the diagonal elements of the matrices. This formula is rather simple when we are on the plane. Namely, assume that the self-affine IFS is given in the formula (2) and let $s_{x}$ and $s_{y}$ be the similarity dimension of the self-similar IFS

$$
\mathcal{H}:=\left\{h_{i}(x):=c_{i} x+u_{i}\right\}_{i=1}^{N} \text { and } \mathcal{V}:=\left\{\varphi_{i}(x):=b_{i} y+v_{i}\right\}_{i=1}^{N}
$$

respectively. Clearly, $\mathcal{H}$ is the horizontal and $\mathcal{V}$ is the vertical projection of the corresponding diagonal system given in the form (1). Let

$$
\widehat{s}_{x}:=\min \left\{s_{x}, 1\right\} \text { and } \widehat{s}_{y}:=\min \left\{s_{y}, 1\right\}
$$

We define $d_{x}$ and $d_{y}$ as the solutions of the following equations:

$$
\sum_{i=1}^{N} c_{i}^{\widehat{s}_{x}} b_{i}^{d_{x}-\widehat{s}_{x}}=1 \text { and } \sum_{i=1}^{N} b_{i}^{\widehat{s}_{y}} c_{i}^{d_{y}-\widehat{s}_{y}}=1 .
$$

Then (c.f. [2, Theorem 4.1]) the affinity dimension of $\mathcal{S}$ is

$$
\operatorname{dim}_{\text {aff }}(\mathcal{S})=\max \left\{d_{x}, d_{y}\right\} .
$$

We say that direction- $x,($ direction- $y)$ dominates if $\operatorname{dim}_{\text {aff }}(\mathcal{S})=d_{x},\left(\operatorname{dim}_{\text {aff }}(\mathcal{S})=d_{y}\right)$ respectively. It follows from the definition of the Hausdorff dimension that the affinity dimension is always an upper bound for the Hausdorff dimension of the attractor (see [10]).

1.4. Notation. Throughout this note, all self-affine IFS on the plane are supposed to be of the form of (2). Without loss of generality we may assume that

$$
S_{i}\left([0,1]^{2}\right) \subset[0,1]^{2} \text { for all } i=1, \ldots, N
$$

As we mentioned above, the attractor of $\mathcal{S}$ is

$$
\Lambda:=\bigcap_{n=1}^{\infty} \bigcup_{\mathbf{i} \in\{1, \ldots, N\}^{n}} S_{\mathbf{i}}\left([0,1]^{2}\right),
$$


where $S_{\mathbf{i}}:=S_{i_{1}} \circ \cdots \circ S_{i_{n}}$ for $\mathbf{i}=\left(i_{1}, \ldots, i_{n}\right)$. Let $\mu$ be the uniform distribution measure on the symbolic space $\Sigma:=\{1, \ldots, N\}^{\mathbb{N}}$. That is $\mu$ is the $(1 / N, \ldots, 1 / N)$-Bernoulli measure. We define $\Pi: \Sigma \rightarrow \Lambda_{\mathcal{S}}$ in the natural way.

$$
\Pi(\mathbf{i}):=\lim _{n \rightarrow \infty} S_{\left.\mathbf{i}\right|_{n}}(0), \quad \mathbf{i} \in \Sigma .
$$

The push forward measure of $\mu$ is $\nu:=\Pi_{*} \mu$. The attractor of the self-similar IFS $\mathcal{H}$ introduced in (4) is denoted by $\Lambda_{\mathcal{H}}$. The natural projection generated by $\mathcal{H}$ is

$$
\Pi_{\mathcal{H}}(\mathbf{i}):=\lim _{n \rightarrow \infty} h_{\left.\mathbf{i}\right|_{n}}(0), \quad \mathbf{i} \in \Sigma .
$$

Clearly, $\Pi_{\mathcal{H}}=\operatorname{proj}_{x} \circ \Pi$, where $\operatorname{proj}_{x}$ is the orthogonal projection to the $x$-axis. The measure on the $x$-axis generated by $\mu$ is

$$
\nu_{x}:=\left(\Pi_{\mathcal{H}}\right)_{*} \mu=\left(\operatorname{proj}_{x}\right)_{*} \nu
$$

Now we introduce the Furstenberg-Kifer measure. The projection $\Pi_{A F}$ below will be used to construct a method to check that the transversality condition holds.

1.5. Furstenberg-Kifer measure. In this subsection we study the action of our system $\mathcal{S}$ on the projective line, in the case

$$
c_{i}>b_{i}, \quad \text {, for all } i=1, \ldots, N .
$$

In particular, the direction- $x$ dominates.

This action can be identified with the action of a simple iterated function system on the line. Consider the vertical line $\xi:=\left\{(1, z) \in \mathbb{R}^{2}: z \in \mathbb{R}\right\}$ on the plane. We can identify $(1, z) \in \xi$ with $\widetilde{z} \in \mathbb{R}$. With this identification we define the self-similar IFS $\mathcal{F}$ on $\xi$ by

$$
\mathcal{F}:=\left\{f_{i}(\widetilde{z}):=\frac{b_{i}}{c_{i}} \widetilde{z}+\frac{d_{i}}{c_{i}}\right\}_{i=1}^{N},
$$

(Recall that in this Subsection $c_{i}>b_{i}$.) It follows from (8) that all $f_{i}$ are strict contractions. So, we can define the natural projection $\Pi_{A F}: \Sigma \rightarrow \xi$ in the usual way:

$$
\Pi_{A F}(\mathbf{i}):=\frac{d_{i_{1}}}{c_{i_{1}}}+\sum_{k=2}^{\infty} \frac{d_{i_{k}}}{c_{i_{k}}} \cdot \prod_{\ell=1}^{k-1} \frac{b_{i_{\ell}}}{c_{i_{\ell}}} .
$$

The importance of $\Pi_{A F}$ is as follows: The action of $\left\{T_{i}\right\}_{i=1}^{N}$ on the projective line is described by the maps $\widetilde{T}_{i}: \xi \rightarrow \xi$

$$
\widetilde{T}_{i}(\widetilde{z}):=\frac{1}{c_{i}} \cdot T_{i} \cdot\left(\begin{array}{l}
1 \\
z
\end{array}\right)
$$

where $\widetilde{z} \in \xi$ is $\widetilde{z}=(1, z)$.

Then

$$
\Pi_{A F}(\mathbf{i})=\widetilde{T}_{i}\left(\Pi_{A F}(\sigma \mathbf{i})\right)
$$

is the natural projection for $\left\{\widetilde{T}_{i}\right\}_{i=1}^{N}$.

As a similar construction have first appeared in [12, we will call the projection under $\Pi_{A F}$ of an ergodic measure $\eta$ defined on $\Sigma$ the Furstenberg-Kifer measure corresponding to $\eta$. 
1.6. The transversality condition. Consider two cylinders $S_{\left.\mathbf{i}\right|_{n}}[0,1]^{2}$ and $S_{\left.\mathbf{j}\right|_{n}}[0,1]^{2}$, $i_{1} \neq j_{1}$. They are parallelograms with two vertical sides. Their angle can be defined as the angle between their non-vertical sides. The following condition holds if any two cylinders $S_{\left.\mathbf{i}\right|_{n}}[0,1]^{2}$ and $S_{\left.\mathbf{j}\right|_{n}}[0,1]^{2}, i_{1} \neq j_{1}$ are either disjoint or have an angle uniformly separated from zero.

Definition 1.1. We say that the diagonally homogeneous IFS S satisfies the transversality condition if is there exists a $K_{3}>0$ such that for every $n$ and for every $\mathbf{i}, \mathbf{j} \in \Sigma$ with $i_{1} \neq j_{1}$ we have

$$
\operatorname{proj}_{x}\left(S_{\left.\mathbf{i}\right|_{n}}[0,1]^{2} \cap S_{\left.\mathbf{j}\right|_{n}}[0,1]^{2}\right)<K_{3} \cdot b^{n} .
$$

Below we present a natural geometric interpretation of this condition which provides a method to check it. First let us define the IFS $\widetilde{\mathcal{S}}$ which acts on $\mathbb{R}^{3}$

$$
\widetilde{\mathcal{S}}:=\left\{\widetilde{S}_{i}(x, y, z):=\left(S_{i}(x, y), \widetilde{T}_{i}(z)\right)\right\}_{i=1}^{N} .
$$

Now we recall two separation properties of IFS. For an IFS $\left\{F_{i}\right\}$ we say that it satisfies the Strong Separation Property (SSP) if its natural projection (in case of $\widetilde{\mathcal{S}}$ it is given by $\left.\mathbf{i} \mapsto\left(\Pi(\mathbf{i}), \Pi_{A F}(\mathbf{i})\right)\right)$ is a bijection. This is equivalent to the existence of a non-empty open set $V$ satisfying

(a): $F_{i}(V) \subset V$ for all $i=1, \ldots, N$ and

(b): $\overline{F_{i}(V)} \cap \overline{F_{j}(V)}=\emptyset$ for all $i \neq j$,

where $\bar{A}$ means the closure of the set $A$.

We can define the Open Set Condition (OSC) in an analogous way: an IFS $\left\{F_{i}\right\}$ satisfies OSC if there exists a non-empty open set $V$ satisfying

(a): $F_{i}(V) \subset V$ for all $i=1, \ldots, N$ and

(b'): $F_{i}(V) \cap F_{j}(V)=\emptyset$ for all $i \neq j$,

\section{Lemma 1.2.}

(i): If $\widetilde{\mathcal{S}}$ satisfies the SSP then the transversality condition holds for $\mathcal{S}$.

(ii): If the transversality condition holds for $\mathcal{S}$ and $S_{i}\left([0,1]^{2}\right) \subset(0,1)^{2}$ for all $i=$ $1, \ldots, N$ then $\widetilde{\mathcal{S}}$ satisfies $S S P$.

Proof. First we prove part (i). We are going to work with long and thin parallelograms. We will call the principal axis of a parallelogram the direction of its long side.

When $\left.\mathbf{i} \mapsto\left(\Pi(\mathbf{i}), \Pi_{A F}(\mathbf{i})\right)\right)$ is a bijection, the usual compactness argument shows that there exists $\ell>0$ such that for any two symbolic sequences $\mathbf{i}, \mathbf{j} \in \Sigma$ with $i_{1} \neq j_{1}$ either

$$
\operatorname{dist}(\Pi(\mathbf{i}), \Pi(\mathbf{j}))>\ell
$$

or

$$
\operatorname{dist}\left(\Pi_{A F}(\mathbf{i}), \Pi_{A F}(\mathbf{j})\right)>\ell .
$$

Take some $N>2(-\log \ell) / \min (-\log c,-\log (b / c))$. In the first case, for $n>N$ the parallelograms $S_{\left.\mathbf{i}\right|_{n}}[0,1]^{2}, S_{\left.\mathbf{j}\right|_{n}}[0,1]^{2}$ do not intersect at all. In the second case, they intersect but transversally (the angle between their their principal axes is larger than $\ell / 2$ ). In both cases (13) holds. This proves the assertion (i).

Now we prove part (ii). Assume now that (13) holds. By the assumption, there exists $\ell>0$ such that $\Lambda \in(\ell, 1-\ell)^{2}$. Fix some large $n$, to be defined later. Fix also some interval $I$ such that $\widetilde{T}_{i}(I) \subset I$ for all $i$.

Consider two words $\left.\mathbf{i}\right|_{n},\left.\mathbf{j}\right|_{n}$ with different first digits $i_{1} \neq j_{1}$. Assume for the moment that the parallelograms $S_{\mathbf{i}_{n}}[\ell / 2,1-\ell / 2]^{2}, S_{\left.\mathbf{j}\right|_{n}}[\ell / 2,1-\ell / 2]^{2}$ intersect each other. Then the parallelogram $S_{\left.\mathbf{i}\right|_{n}}[0,1]^{2}$ must intersect an internal point of parallelogram $S_{\left.\mathbf{j}\right|_{n}}[0,1]^{2}$, 
a point which is in distance at least $\ell b^{n} / 2$ from the vertical boundary and in distance at least $\ell c^{n} / 2$ from the horizontal (non-vertical) boundary of $S_{\left.\mathbf{j}\right|_{n}}[0,1]^{2}$. Hence, if (13) holds then the angle between the principal axes of the parallelograms is at least $K_{4}=\ell / 2 K_{3}$. In particular, if $n$ was so large that $(b / c)^{n}|I|<K_{4} / 2$ then

$$
\widetilde{S}_{\left.\mathbf{i}\right|_{n}}\left([\ell, 1-\ell]^{2} \times I\right) \cap \widetilde{S}_{\left.\mathbf{j}\right|_{n}}\left([\ell, 1-\ell]^{2} \times I\right)=\emptyset .
$$

On the other hand, when the parallelograms $S_{\left.\mathbf{i}\right|_{n}}[\ell / 2,1-\ell / 2]^{2}, S_{\left.\mathbf{j}\right|_{n}}[\ell / 2,1-\ell / 2]^{2}$ do not intersect each other then (15) also holds. Hence, it holds for all pairs of words $\left.\mathbf{i}\right|_{n},\left.\mathbf{j}\right|_{n}$ with different first digits. This implies that $\widetilde{S}$ satisfies SSP for the set

$$
V=\bigcup_{\left.\mathbf{k}\right|_{n-1} \in\{1, \ldots, N\}^{n-1}} \widetilde{S}_{\left.\mathbf{k}\right|_{n-1}}\left((\ell, 1-\ell)^{2} \times I^{o}\right) .
$$

This is complete the proof of part (ii) of the assertion.

\subsection{Lyapunov exponents, Lyapunov dimension, projection entropy and expo- nential separation condition.}

Notation 1.3. Let $\Psi=\left\{\psi_{i}\right\}_{i=1}^{N}$ be strictly contracting IFS on $\mathbb{R}^{d}$. Let $\Sigma=\{1, \ldots, N\}^{\mathbb{N}}$ be the symbolic space, $\sigma$ the left-shift operator on $\Sigma$ and the natural projection is $\Pi\left(i_{1}, i_{2}, \ldots\right)=$ $\lim _{n \rightarrow \infty} \psi_{i_{1}} \circ \cdots \circ \psi_{i_{n}}(\underline{0})$. Let $\mathbf{p}:=\left(p_{1}, \ldots, p_{N}\right)$ be a probability vector. This generates a Bernoulli measure on $\Sigma$, which is denoted by $\mathbb{P}_{\mathbf{p}}:=\left\{p_{1}, \ldots, p_{N}\right\}^{\mathbb{N}}$. The corresponding self-similar measure is

$$
\nu_{\Psi, \mathbf{p}}:=\Pi_{*} \mathbb{P}_{\mathbf{p}}
$$

Finally, for every $n \geq 1$ we put

$$
\Sigma_{n}:=\{1, \ldots, N\}^{n} \text { and } \Sigma_{*}:=\bigcup_{n=1}^{\infty} \Sigma_{n} .
$$

1.7.1. Lyapunov exponents and Lyapunov dimension. The general definition of Lyapunov exponents can be found e.g. in [27]. However, in the special case of systems generated by lower triangular matrices like in (2) the vertical direction is preserved by the system. Hence, the Lyapunov exponents can be expressed by the diagonal elements only [8].

The Lyapunov dimension of a self-affine measure was introduced in [16, Definition 3]. It is always an upper bound on the Hausdorff dimension of the measure. In the special cases we consider in this note, we can write down a simpler formula for the Lyapunov dimension of $\nu_{\Psi, \mathbf{p}}$ :

Example 1.4. (a): If $\Psi$ is a self-similar IFS on the line then $\nu_{\Psi, \mathbf{p}}$ has one Lyapunov exponent which is defined as

$$
\chi_{\Psi, \mathbf{p}}:=-\sum_{i=1}^{N} p_{i} \cdot \log \left|\psi^{\prime}(0)\right| .
$$

(b): Assume that $\Psi=\mathcal{S}^{\text {diag }}$ is a diagonally self-affine IFS on the plane defined as in (1). Then its horizontal and vertical projections are self-similar IFSs $\mathcal{H}$ and $\mathcal{V}$ on the line of the form (4). In this case, $\nu_{\Psi, \mathbf{p}}$ has two (not necessarily distinct) Lyapunov exponents: $\chi_{\mathcal{H}, \mathbf{p}}$ and $\chi_{\mathcal{V}, \mathbf{p}}$. We say that direction- $x$ dominates if the following counter intuitive condition holds:

$$
\chi_{\mathcal{H}, \mathbf{p}} \leq \chi_{\mathcal{V}, \mathbf{p}}
$$


Assuming for example that direction-x dominates, the Lyapunov dimension can be expressed as

$$
D\left(\nu_{\Psi, \mathbf{p}}\right)= \begin{cases}\frac{h\left(\mathbb{P}_{\mathbf{p}}\right)}{\chi_{\mathcal{H}, \mathbf{p}}}, & \text { if } ; h\left(\mathbb{P}_{\mathbf{p}}\right) \leq \chi_{\mathcal{H}, \mathbf{p}} \\ \frac{h\left(\mathbb{P}_{\mathbf{p}}\right)+\chi_{\mathcal{V}, \mathbf{p}}-\chi_{\mathcal{H}, \mathbf{p}}}{\chi_{\mathcal{V}, \mathbf{p}}}, & \text { if } ; \chi_{\mathcal{H}, \mathbf{p}}<h\left(\mathbb{P}_{\mathbf{p}}\right) \leq \chi_{\mathcal{H}, \mathbf{p}}+\chi_{\mathcal{V}, \mathbf{p}} \\ 2 \frac{h\left(\mathbb{P}_{\mathbf{p}}\right)}{\chi_{\mathcal{H}, \mathbf{p}}+\chi_{\mathcal{V}, \mathbf{p}}}, & \text { otherwise. }\end{cases}
$$

(c): The formulas (20) depend only on the entropy and the Lyapunov exponents, which are depending on the diagonal elements only. In the general lower triangular case when $\Psi=\mathcal{S}$ given by the formula (2), the non-diagonal element of the matrix $A_{i_{0}} \cdots A_{i_{n}}$ has the form

$$
d_{i_{0}, \ldots, i_{n}}=\sum_{k=0}^{n} d_{i_{k}}\left(\prod_{j=k+1}^{n} c_{i_{j}}\right)\left(\prod_{j=0}^{k-1} b_{i_{j}}\right) .
$$

Thus, by the ergodicity, simple algebraic manipulation show that

$$
\liminf _{n \rightarrow \infty} \frac{-\log \left|d_{i_{0}, \ldots, i_{n}}\right|}{n} \geq \chi_{\mathcal{H}, \mathbf{p}} \text { almost surely. }
$$

Hence, the Lyapunov dimension depends only on the diagonal elements of the matrices, so the equation (20) still holds.

1.7.2. Projection entropy. We recall the definition of the projective entropy, which was introduced by Feng and $\mathrm{Hu}[11$.

Here we use Notation 1.3 . That is let $\Psi=\left\{\psi_{i}\right\}_{i=1}^{N}$ be a strictly contracting IFS on $\mathbb{R}^{d}$. Moreover, let $\mathfrak{m}$ be a $\sigma$-invariant ergodic measure on $\Sigma$. Let $\mathcal{P}=\{[1], \ldots,[N]\}$ be the partition of $\Sigma$, where $[i]=\left\{\mathbf{i} \in \Sigma: i_{1}=i\right\}$ and we write $\mathcal{B}$ for the Borel $\sigma$-algebra of $\mathbb{R}^{d}$. Feng and $\mathrm{Hu}$ [11, Definition 2.1] defined the projection entropy of $\mathfrak{m}$ under $\Pi$ with respect to $\Psi$

$$
h_{\Pi}(\mathfrak{m}):=H_{\mathfrak{m}}\left(\mathcal{P} \mid \sigma^{-1} \Pi^{-1} \mathcal{B}\right)-H_{\mathfrak{m}}\left(\mathcal{P} \mid \Pi^{-1} \mathcal{B}\right),
$$

where $H_{\mathfrak{m}}(\xi \mid \eta)=-\int \log \mathfrak{m}_{x}^{\eta}(\xi(x)) d \mathfrak{m}(x)$ denotes the usual conditional entropy of $\xi$ given $\eta$, where $\mathfrak{m}_{x}^{\eta}$ denotes the conditional measure of $\mathfrak{m}$ supported on $\eta(x)$ (the element of the partition $\eta$, which contains $x$ ).

1.7.3. Hochman's exponential separation condition. Hochman introduced the following Diophantine-type condition in [13].

Let $\Psi=\left\{\psi_{i}\right\}_{i=1}^{N}$ be a self-similar IFS on $\mathbb{R}$. Let $\Delta_{n}(\psi)$ be the minimum of $\Delta(\bar{\imath}, \bar{\jmath})$ for distinct $\bar{\imath}, \bar{\jmath} \in \Sigma_{n}$, where

$$
\Delta(\bar{\imath}, \bar{\jmath})=\left\{\begin{array}{cc}
\infty & \psi_{\bar{\imath}}^{\prime}(0) \neq \psi_{\bar{\jmath}}^{\prime}(0) \\
\left|\psi_{\bar{\imath}}(0)-\psi_{\bar{\jmath}}(0)\right| & \psi_{\bar{\imath}}^{\prime}(0)=\psi_{\bar{\jmath}}^{\prime}(0) .
\end{array}\right.
$$

Condition. We say that the self-similar IFS $\psi$ satisfies Hochman's exponential separation condition if there exists an $\varepsilon>0$ and an $n_{k} \uparrow \infty$ such that

$$
\Delta_{n_{k}}>\varepsilon^{n_{k}} \text {. }
$$

We remark that in our earlier paper [6, p.2] we stated this condition in an unnecessarily strict way. Namely, on [6, p.2] we required that (21) holds for all elements of the sequence $\left\{\Delta_{n}\right\}_{n=1}^{\infty}$, while it is enough that this inequality holds only on a subsequence as stated above. However, all the assertions of [6] remain valid under the weaker condition (21) since in [6] we never used the condition $\Delta_{n}>\varepsilon^{n}$ directly, we used only the conclusions of Hochman's Theorems [13]. 


\section{Theorems of Hochman and Feng, Hu}

Hochman proved the following very important assertion in [13, Theorem 1.1].

Theorem 2.1 (Hochman). Here we use Notation 1.3. Let $\Psi=\left\{\psi_{i}\right\}_{i=1}^{N}$ be a self-similar IFS on the real line. Assume that $\Psi$ satisfies the Hochman's exponential separation condition. Let $\mathbf{p}=\left(p_{1}, \ldots, p_{N}\right)$ be an arbitrary probability vector.

Then

$$
\operatorname{dim}_{H}\left(\nu_{\Psi, \mathbf{p}}\right)=\min \left\{1, \frac{h\left(\mathbb{P}_{\mathbf{p}}\right)}{\chi_{\Psi, \mathbf{p}}}\right\}
$$

where $h\left(\mathbb{P}_{\mathbf{p}}\right)=-\sum_{i=1}^{N} p_{i} \log p_{i}$.

The ratio on the right hand side of 22 is the similarity dimension of $\nu_{\Psi, \mathbf{p}}$. That is

$$
\operatorname{dim}_{\mathrm{S}}\left(\nu_{\Psi, \mathbf{p}}\right):=\frac{h\left(\mathbb{P}_{\mathbf{p}}\right)}{\chi_{\Psi, \mathbf{p}}} .
$$

We note that the similarity dimension is a special case of Lyapunov dimension, if the affinity transformations are similarity transformations.

For the next two theorems we refer to [11, Theorem 2.8] and [11, Theorem 2.11].

Theorem 2.2 (Feng, Hu). Here we use Notation 1.3 and notation from Section 1.7.2. Let $\Psi$ be a self-similar IFS on the line and $\mathbf{p}$ be a probability vector. Then

$$
\operatorname{dim}_{H}\left(\nu_{\Psi, \mathbf{p}}\right)=\frac{h_{\Pi}\left(\mathbb{P}_{\mathbf{p}}\right)}{\chi_{\Psi, \mathbf{p}}} .
$$

If we put Theorem 2.1 together with Theorem 2.2 we obtain

Corollary 2.3. Let $\Psi$ be a self-similar IFS on the line which satisfies the Hochman's exponential separation condition. Then $h_{\Pi}\left(\mathbb{P}_{\mathbf{p}}\right)=\min \left\{h\left(\mathbb{P}_{\mathbf{p}}\right), \chi_{\Psi, \mathbf{p}}\right\}$.

Now we consider the diagonally self-affine case on the plane.

Theorem 2.4 (Feng, Hu). Given the diagonally self-affine IFS like in (1). We assume that it satisfies the SSP. Its horizontal and vertical parts (see (4)) are denoted by $\mathcal{H}$ and $\mathcal{V}$.

Fix a probability vector $\mathbf{p}=\left(p_{1}, \ldots, p_{N}\right)$. We consider the Lyapunov exponents $\chi_{\mathcal{H}, \mathbf{p}}$ and $\chi_{\mathcal{V}, \mathbf{p}}$ as in (18). Without loss of generality we assume that direction-x dominates, which means that

holds. Then

$$
0<\chi_{\mathcal{H}, \mathbf{p}} \leq \chi_{\mathcal{V}, \mathbf{p}}
$$

$$
\operatorname{dim}_{\mathrm{H}}\left(\nu_{\mathcal{S}^{\text {diag }}, \mathbf{p}}\right)=\frac{h\left(\mathbb{P}_{\mathbf{p}}\right)}{\chi_{\mathcal{V}, \mathbf{p}}}+\left(1-\frac{\chi_{\mathcal{H}, \mathbf{p}}}{\chi_{\mathcal{V}, \mathbf{p}}}\right) \cdot \operatorname{dim}_{\mathrm{H}}\left(\nu_{\mathcal{H}, \mathbf{p}}\right)
$$

Observe that the Hausdorff dimension of the measure $\nu_{\mathcal{S} \text { diag, }}$ is equal to its Lyaponov dimension if and only if $\operatorname{dim}_{\mathrm{H}}\left(\nu_{\mathcal{H}, \mathbf{p}}\right)=1$.

\section{THE TRIVIAL CASE AND FURTHER NOTATION}

\subsection{The trivial case.}

Lemma 3.1. Assume that direction-x dominates and $\sum_{i=1}^{N}\left|c_{i}\right|<1$. Further, we assume that the exponential separation condition holds for the IFS $\mathcal{H}$ defined in (4). Then $\operatorname{dim}_{\mathrm{H}} \Lambda_{\mathcal{S}}=$ $s_{x}$. 
The proof is immediate since in this case $\operatorname{dim}_{\text {aff }}(\mathcal{S})=s_{x}$ which is an upper bound always. The lower estimate comes from Hochman Theorem: the dimension of the attractor of $\mathcal{H}$ is equal to $s_{x}$ and this attractor is a projection of $\Lambda$. Apart from the trivial case, we can get results only if we assume that

$$
c:=c_{i} \text { and } b:=b_{i} \text { holds for all } i .
$$

3.2. Further notation. We use the notation of Section 1.4 .

That is, from now on we always assume that the matrices $T_{i}$ in (2) are of the form

$$
T_{i}=\left(\begin{array}{cc}
c & 0 \\
d_{i} & b
\end{array}\right)
$$

Clearly, if $c>b$ then direction- $x$ dominates and if $b>c$ then direction- $y$ dominates.

We use the notation of Section 1.4, where we introduced the uniformly distributed Bernoulli measure $\mu$ on the symbolic space $\Sigma$, the natural projection $\Pi$, the projection of $\mu$ to the attractor $\Lambda \subset \mathbb{R}^{2}$ was called $\nu$ and the projection of $\nu$ to the $x$-axis was denoted by $\nu_{x}$.

The measures $\mu$ and $\nu$ can be disintegrated, according to the partitions

$$
\begin{gathered}
\xi_{a}^{s}:=\left\{\mathbf{i} \in \Sigma: \Pi_{\mathcal{H}(\mathbf{i})}=a\right\} \text { and } \xi_{a}:=\{(x, y): x=a, y \in[0,1]\} \\
\mu\left(A^{s}\right)=\int \alpha_{a}^{\mu}(A) d \mu_{x}(a), \quad \nu(A)=\int \alpha_{a}^{\nu}(A) d \nu_{x}(a),
\end{gathered}
$$

for any sets $A^{s} \subset \Sigma$ and $A \subset[0,1]^{2}$. That is the probability measures $\alpha_{a}^{\mu}$ and $\alpha_{a}^{\nu}$ are supported by $\xi_{a}^{s}$ and $\xi_{a}$ respectively.

Clearly,

$$
\mu_{x}=\left(\operatorname{proj}_{x}\right)_{*} \nu=\nu_{x} .
$$

For an arbitrary $a \in[0,1]$ we write $\Pi_{a}$ for the restriction of the natural projection $\Pi$ to $\xi_{a}^{s}$. That is $\Pi_{a}: \xi_{a}^{s} \cap \Sigma \rightarrow \xi_{a} \cap \Lambda_{\mathcal{S}}$

Then we have

$$
\left(\Pi_{a}\right)_{*} \alpha_{a}^{\mu}=\alpha_{a}^{\nu}
$$

Now we introduce the vertical distance, vertical ball and and vertical neighborhood of a set:

$$
\begin{gathered}
\operatorname{dist}_{y}\left(\left(x_{0}, y_{0}\right),(x, y)\right):= \begin{cases}\left|y-y_{0}\right|, & \text { if } x=x_{0} ; \\
\infty, & \text { otherwise, }\end{cases} \\
B_{y}\left(\left(x_{0}, y_{0}\right), r\right):=\left\{(x, y): x=x_{0} \text { and }\left|y-y_{0}\right|<r\right\} .
\end{gathered}
$$

Further, let

$$
U_{y}(H, r):=\bigcup_{\left(x_{0}, y_{0}\right) \in H} B_{y}\left(\left(x_{0}, y_{0}\right), r\right) .
$$

We define the vertical distance in the symbolic space as well:

$$
\operatorname{dist}_{y}^{s}(\mathbf{i}, \mathbf{j}):= \begin{cases}b^{|\mathbf{i} \wedge \mathbf{j}|}, & \text { if } \Pi_{\mathcal{H}}(\mathbf{i})=\Pi_{\mathcal{H}}(\mathbf{j}) ; \\ \infty, & \text { otherwise, }\end{cases}
$$

$B_{y}^{s}(\mathbf{i}, r)$ and $U_{y}^{s}\left(H^{s}, r\right)$ are then defined analogously to $B_{y}((x, y), r)$ and $U_{y}(H, r)$. 


\section{Direction- $x$ DOMinates}

In this case by (6),

$$
\operatorname{dim}_{\mathrm{aff}} \mathcal{S}= \begin{cases}\frac{\log N}{-\log c}, & \text { if } N c<1 \\ 1+\frac{\log (N c)}{-\log b}, & \text { if } N c>1\end{cases}
$$

As we have seen above the $N c<1$ case is obvious. So, from now on we may assume that $N c>1$.

Consider the iterated function system $\mathcal{H}$. If $N c>1$ then the affinity dimension of this system is larger than 1 . For systems like that there are many results proving (under different assumptions) that their natural measure is not only absolutely continuous but also has $L^{q}$ density for some $q>1$. For example, for Bernoulli convolutions the natural measure has $L^{2}$ density for almost all parameters ([25]), has $L^{q}$ density for some $q>1$ for all parameters except a dimension zero subset ([22]), and by the new preprint of Shmerkin it has $L^{q}$ density for all $q>1$ for all parameters except a dimension zero subset ([21]). It turns out that the knowledge of $L^{q}$ properties of the density of natural measure is quite useful.

We are going to use the following assumptions:

\section{Assumption A.}
(A1) $c>\frac{1}{N}$
(A2) $b<\frac{1}{N}$
(A3) $\nu_{x} \ll \mathcal{L}$ eb with $L^{q}$ density, for some $q>1$,
(A4) $\mathcal{S}$ satisfies transversality. (Definition 1.1.)

When we replace (A3) with a stronger assumption (B3), we relax our assumption about $b$ :

\section{Assumption B.}

(B1) $c>\frac{1}{N}$

(B2) $b<c$.

(B3) $\nu_{x} \ll \mathcal{L}$ eb with $L^{q}$ density, for all $q>1$,

(B4) $\mathcal{S}$ satisfies transversality.

If we consider the corresponding diagonal system (the one in (1D) then assumptions (A1, B1) say that the similarity dimension to the $x$-axis is greater than one in both cases, while (A2) postulates that the projection to the vertical axis of the corresponding diagonal system has similarity dimension less than one.

Our new results when $c>b$ are as follows:

Theorem 4.1. Let $\mathcal{S}$ be a self-affine IFS of the form (2) satisfying Assumption A. Then

$$
\operatorname{dim}_{\mathrm{H}}(\Lambda)=\operatorname{dim}_{\mathrm{H}}(\nu)=1+\frac{\log (N c)}{-\log b} .
$$

Theorem 4.2. Let $\mathcal{S}$ be a self-affine IFS of the form (2) satisfying Assumption B. Then

$$
\operatorname{dim}_{\mathrm{H}}(\Lambda)=\operatorname{dim}_{\mathrm{H}}(\nu)=\min \left(2,1+\frac{\log (N c)}{-\log b}\right) .
$$

Observe that Assumption A guarantees that $1+\log (N c) /-\log b<2$.

4.1. The proof of Theorem 4.1. First we give the proof assuming a density assertion (Proposition 4.3). The proof of Proposition 4.3 is given in Sections 4.1.2 and 4.1.3. 
4.1.1. The proof of Theorem 4.1 modulo a density lemma.

Proof of Theorem 4.1. Let $h_{x}(\mu)$ be the projection entropy which corresponds to the projection $\Pi_{\mathcal{H}}$ (that is, the entropy of $\left(\mathcal{H}, \Pi_{\mathcal{H}}(\mu)\right)$ ). It follows from [11, Proposition 4.14] that

$$
-\lim _{n \rightarrow \infty} \frac{\log \alpha_{\Pi_{\mathcal{H}}(\mathbf{i})}^{\mu}\left(B_{y}^{s}\left(\mathbf{i}, b^{n}\right)\right)}{n}=h(\mu)-h_{x}(\mu) \text { for } \mu \text { a.a.i. }
$$

Observe that $h(\mu)=\log N$. On the other hand, by Assumption (A3), it follows from Corollary 2.3 that we have

$$
h_{x}(\mu)=-\log c .
$$

Putting these together we obtain that

$$
-\lim _{n \rightarrow \infty} \frac{\log \alpha_{\Pi_{\mathcal{H}}(\mathbf{i})}^{\mu}\left(B_{y}^{s}\left(\mathbf{i}, b^{n}\right)\right)}{n}=\log (N c) \text { for } \mu \text { a.a.i. }
$$

Our aim below is to prove the corresponding statement for $\alpha_{a_{0}}^{\nu}$ :

$$
-\lim _{n \rightarrow \infty} \frac{\log \alpha_{a_{0}}^{\nu}\left(B_{y}\left(\Pi_{a_{0}}\left(y_{0}\right), b^{n}\right)\right)}{n}=\log \left(N_{c}\right) \text { for } \nu \text { a.a. }\left(a_{0}, y_{0}\right) \text {. }
$$

Namely, if Proposition 4.3 holds then (37) follows and this implies that for $\nu_{x}$-a.a. $a_{0} \in$ $[0,1]$ we have

$$
\operatorname{dim}_{\mathrm{H}}\left(\Lambda_{\mathcal{S}} \cap \xi_{a_{0}}\right) \geq \frac{\log (N c)}{-\log b} .
$$

Using that $\nu_{x}$ is absolutely continuous w.r.t Lebesgue measure, we obtain that (38) holds for a set of Hausdorff dimension 1 of $a_{0}$. Then by [9, Theorem 5.8] we obtain that

$$
\operatorname{dim}_{\mathrm{H}} \Lambda_{\mathcal{S}} \geq 1+\frac{\log (N c)}{-\log b} .
$$

The opposite inequality is immediate since $1+\frac{\log (N c)}{-\log b}$ is the affinity dimension of $\mathcal{S}$ which is always an upper bound on the Hausdorff dimension of the attractor. So, to complete the proof of Theorem 4.1 it is enough to verify Proposition 4.3 below.

4.1.2. Density in the symbolic space versus on the attractor.

Proposition 4.3. For $\mu$-a.a. i we have

$$
\liminf _{n \rightarrow \infty} \frac{1}{n} \log \alpha_{\Pi_{\mathcal{H}}(\mathbf{i})}^{\mu}\left(B_{y}^{s}\left(\mathbf{i}, b^{n}\right)\right)=\liminf _{n \rightarrow \infty} \frac{1}{n} \log \alpha_{\Pi_{\mathcal{H}}(\mathbf{i})}^{\nu}\left(B_{y}\left(\Pi(\mathbf{i}), b^{n}\right)\right) .
$$

The same holds if we replace liminf with lim sup on both sides.

We need the following notion:

Definition 4.4 (Definition of $L$ ). Let $\mathbf{i} \in \Sigma$ and we write

$$
Z_{n}(\mathbf{i}):=\left\{\mathbf{j}:\left.\mathbf{j}\right|_{n} \neq\left.\mathbf{i}\right|_{n}, \Pi_{\mathcal{H}}(\mathbf{j})=\Pi_{\mathcal{H}}(\mathbf{i})\right\}=\left[\left.\mathbf{i}\right|_{n}\right]^{c} \cap \Pi_{\mathcal{H}}^{-1}\left(\Pi_{\mathcal{H}}(\mathbf{i})\right),
$$

where $\left[\left.\mathbf{i}\right|_{n}\right]:=\left\{\mathbf{j} \in \Sigma:\left.\mathbf{i}\right|_{n}=\left.\mathbf{j}\right|_{n}\right\}$, and for a set $A$, we write $A^{c}$ for the complement of $A$. We define the function $L$ which is the vertical distance from the closest point having a different first cylinder in its symbolic representation:

$$
L(\mathbf{i}):=\min \left\{\operatorname{dist}_{y}(\mathbf{i}, \mathbf{j}) ; \mathbf{j} \in Z_{1}(\mathbf{i})\right\} .
$$


We say that an $\mathbf{i} \in \Sigma$ is $\varepsilon$-good if $\exists C=C(\mathbf{i}, \varepsilon)>0$ such that for all $n \geq 0$ we have $L\left(\sigma^{n} \mathbf{i}\right) \geq C \cdot \mathrm{e}^{-\varepsilon n}$. Moreover, $\mathbf{i} \in \Sigma$ is called $\operatorname{good}$ if it is $\varepsilon$-good for all $\varepsilon>0$. That is, we write

$$
G:=\{\mathbf{i} \in \Sigma: \mathbf{i} \text { is } \varepsilon \text {-good for all } \varepsilon>0\} .
$$

The geometric meaning of $G$ is given by following observation.

Claim 1. Assume that $\mathbf{i} \in \Sigma$ is $\varepsilon$-good for some $C, \varepsilon>0$. Let $\boldsymbol{\omega} \in\{1, \ldots, N\}^{n}$ such that $\boldsymbol{\omega} \neq\left.\mathbf{i}\right|_{n}$. Then

$$
\Lambda_{\boldsymbol{\omega}} \cap B_{y}\left(\Pi(\mathbf{i}), C \cdot b^{n} e^{-\varepsilon n}\right)=\emptyset .
$$

Proof. Let $k:=|\boldsymbol{\omega} \wedge \mathbf{i}|<n$. By assumption we have

$$
\operatorname{dist}_{y}\left(\Pi\left(\sigma^{k} \mathbf{i}\right), \Lambda_{\omega_{k+1}}\right) \geq C \cdot \mathrm{e}^{-\varepsilon k} .
$$

Using that $S_{\left.\mathbf{i}\right|_{k}}=S_{\left.\boldsymbol{\omega}\right|_{k}}, \Pi(\mathbf{i})=S_{\left.\mathbf{i}\right|_{k}}\left(\Pi\left(\sigma^{k} \mathbf{i}\right)\right)$ and $S_{\left.\boldsymbol{\omega}\right|_{k}}\left(\Lambda_{\omega_{k+1}}\right)=\Lambda_{\left.\boldsymbol{\omega}\right|_{k+1}} \supset \Lambda_{\boldsymbol{\omega}}$ the statement follows from the fact that $S_{\left.\mathbf{i}\right|_{k}}$ contracts in vertical direction by factor $b^{k}$.

Lemma 4.5. Let $\mathbf{i} \in G$ and $\Pi(\mathbf{i})=\left(a_{0}, y_{0}\right)$. Let $\eta^{s}$ be a measure on $\xi_{a_{0}}^{s}$ and we write $\eta:=(\Pi)_{*} \eta^{s}$. Then

$$
\liminf _{n \rightarrow \infty} \frac{1}{n} \log \eta^{s}\left(B_{y}^{s}\left(\mathbf{i}, b^{n}\right)\right)=\liminf _{n \rightarrow \infty} \frac{1}{n} \log \eta\left(B_{y}\left(\Pi(\mathbf{i}), b^{n}\right)\right) .
$$

The equality stays true if we replace liminf by limsup on both sides.

Proof. Clearly, $\eta$ is supported on $\xi_{a_{0}}$. The direction " $\leq "$ follows from the fact that $\Pi$ restricted to $\xi_{a_{0}}^{s}$ is a Lipschitz map (with Lipschitz constant 1). This is true for every $a_{0}$, hence the inequality in this direction is true for all $\mathbf{i} \in \Sigma$.

Now we verify the " $\geq$ " part. Fix an $\varepsilon>0$ an $n$ and an $\mathbf{i} \in \Sigma$ which is $\varepsilon$-good with a constant $C>0$.

As $\mathbf{i}$ is $\varepsilon$-good, by 44 ,

$$
\eta\left(B_{y}\left(\Pi(\mathbf{i}), C \cdot b^{n} \mathrm{e}^{-\varepsilon n}\right)\right) \leq \eta^{s}\left(\Pi(\mathbf{i}), b^{n}\right) .
$$

Passing with $n$ to infinity, we get

$$
\left(1-\frac{\varepsilon}{\log b}\right) \liminf _{n \rightarrow \infty} \frac{1}{n} \log \eta^{s}\left(B_{y}^{s}\left(\mathbf{i}, b^{n}\right)\right) \geq \liminf _{n \rightarrow \infty} \frac{1}{n} \log \eta\left(B_{y}\left(\Pi(\mathbf{i}), b^{n}\right)\right) .
$$

As $\varepsilon>0$ can be chosen arbitrarily small, the assertion follows.

4.1.3. $\mu$-almost every point is good. Our goal is to prove

Proposition 4.6. Let $\mu$ be the uniform distribution on $\Sigma$ and $G \subset \Sigma$ be defined by (43). Then

$$
\mu(G)=1
$$

Assuming this, we obtain

Proof of Proposition 4.3. The proof immediately follows from Lemma 4.5 and Proposition 4.6 .

Recall that, as we discussed above, the proof of Theorem 4.1 follows from Proposition 4.3. So the only thing left is to prove Proposition 4.6. To do so we need the following Lemma. First we introduce

$$
V_{\varepsilon, n}:=\left\{\mathbf{j}: L(\mathbf{j})<\mathrm{e}^{-\varepsilon n}\right\}
$$


Lemma 4.7. There exists a constant $K_{4}>0$ an $r \in(0,1)$ such that for all $n \geq 0$.

$$
\mu\left(V_{\varepsilon, n}\right)<K_{4} \cdot r^{n} .
$$

Proof. Let $\ell:=\frac{\varepsilon}{-\log b} \cdot n$. Then $b^{\ell}=\mathrm{e}^{-\varepsilon n}$. In the rest of this proof we always suppose that

$$
\omega, \tau \in \Sigma_{\ell}, \quad \omega_{1} \neq \tau_{1} .
$$

Recall that by assumption there exist a $q>1$ such that $M:=\int \varphi^{q}(t) d t<\infty$, where $\varphi(t)$ is the density function of $\nu_{x}$. It follows from Assumption (A2) that we can choose a $\delta \in(0,1)$ such that

$$
N b \cdot(N c)^{2 \delta}<1
$$

and let

$$
\operatorname{Bad}_{\ell, \delta}^{1}:=\left\{t \in[0,1]: \varphi(t)>(N c)^{\delta \ell}\right\}
$$

and

$$
\operatorname{Bad}_{\ell, \delta}^{2}:=\left\{t \in[0,1]: \sum_{|\boldsymbol{\tau}|=\ell} \mathbb{1}_{h_{\boldsymbol{\tau}}([0,1])}(t)>(N c)^{(1+\delta) \ell}\right\}
$$

It follows from Corollary 7.5 of the Appendix that

$$
\nu_{x}\left(\operatorname{Bad}_{\ell, \delta}^{2}\right) \leq K_{6} \cdot(N c)^{-\ell \delta(q-1)},
$$

where $K_{6}>0$ is a constant. Furthermore by Markov inequality

$$
M=\int_{0}^{1} \varphi^{q}(t) d t=\int_{0}^{1} \varphi^{q-1}(t) d \nu_{x}(t) \geq \nu_{x}\left(\operatorname{Bad}_{\ell, \delta}^{1}\right) \cdot(N c)^{(q-1) \delta \ell} .
$$

that is

$$
\nu_{x}\left(\operatorname{Bad}_{\ell, \delta}^{1}\right) \leq M \cdot(N c)^{-\delta \ell(q-1)}
$$

Now we define

$$
I_{\boldsymbol{\omega}, \boldsymbol{\tau}}:=\operatorname{proj}_{x}\left(S_{\boldsymbol{\omega}}\left([0,1]^{2}\right) \cap U_{y}\left(S_{\boldsymbol{\tau}}\left([0,1]^{2}\right), b^{\ell}\right)\right) \backslash \operatorname{Bad}_{\ell, \delta}^{2} .
$$

We define

$$
R_{\boldsymbol{\omega}, \boldsymbol{\tau}}:=\left(I_{\boldsymbol{\omega}, \boldsymbol{\tau}} \times[0,1]\right) \cap S_{\boldsymbol{\omega}}\left([0,1]^{2}\right) .
$$

That is $R_{\boldsymbol{\omega}, \boldsymbol{\tau}}$ consist of those elements of $S_{\boldsymbol{\omega}}$ which are "bad" because of $S_{\boldsymbol{\tau}}\left([0,1]^{2}\right)$. It follows from transversality (Definition 1.1) that

$$
\left|I_{\omega, \tau}\right|<3 K_{3} \cdot b^{\ell}
$$

Let

$$
\widetilde{R}_{\boldsymbol{\omega}, \boldsymbol{\tau}}:=[\boldsymbol{\omega}] \cap \Pi^{-1}\left(R_{\boldsymbol{\omega}, \boldsymbol{\tau}}\right) .
$$

The importance of $\widetilde{R}_{\boldsymbol{\omega}, \tau}$ is that it follows from elementary geometry that

$$
\left\{\mathbf{j} \in[\boldsymbol{\omega}]: \operatorname{proj}_{x}(\mathbf{j}) \notin \operatorname{Bad}_{\ell, \delta}^{2}, \exists \mathbf{i} \in \boldsymbol{\tau}, \operatorname{dist}_{y}(\Pi(\mathbf{i}), \Pi(\mathbf{j}))<b^{\ell}\right\} \subset \widetilde{R}_{\boldsymbol{\omega}, \boldsymbol{\tau}}
$$

It follows from the construction of $R_{\boldsymbol{\omega}, \tau}$ that there is a (possibly empty) interval $J_{\boldsymbol{\omega}, \tau}$ such that

$$
S_{\boldsymbol{\omega}}^{-1}\left(R_{\boldsymbol{\omega}, \boldsymbol{\tau}}\right)=\left(J_{\boldsymbol{\omega}, \boldsymbol{\tau}} \backslash h_{\boldsymbol{\omega}}^{-1}\left(\operatorname{Bad}_{\ell, \delta}^{2}\right)\right) \times[0,1]
$$




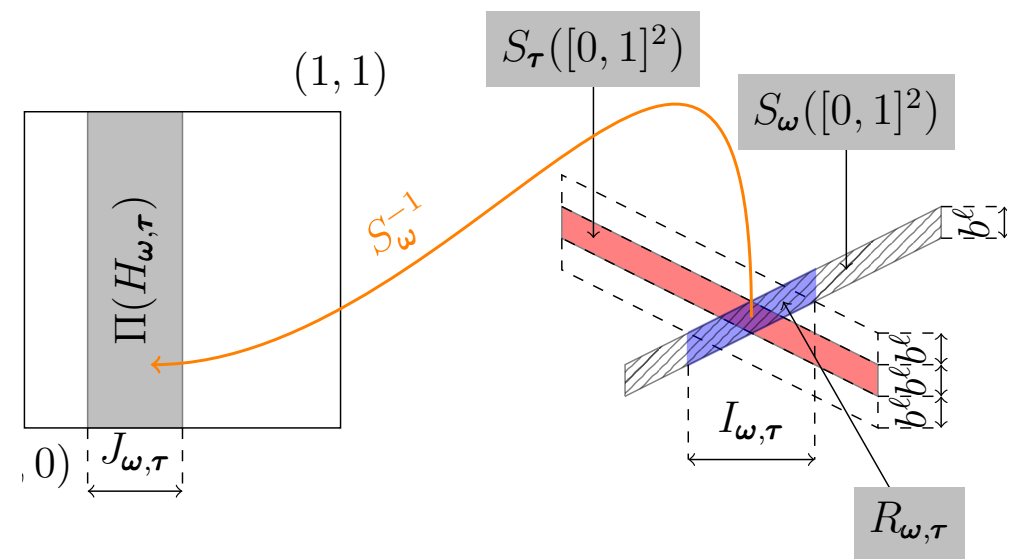

Figure 1. The idea of proof of Lemma 4.7. $\left(\operatorname{Bad}_{\ell, \delta}^{1}, \operatorname{Bad}_{\ell, \delta}^{2}\right.$ not included.)

where the length of the interval

$$
\left|J_{\omega, \tau}\right| \leq 3 K_{3}\left(\frac{b}{c}\right)^{\ell}
$$

Let

$$
H_{\boldsymbol{\omega}, \boldsymbol{\tau}}:=\Pi^{-1}\left(\left(J_{\boldsymbol{\omega}, \boldsymbol{\tau}} \backslash h_{\boldsymbol{\omega}}^{-1}\left(\operatorname{Bad}_{\ell, \delta}^{2}\right)\right) \times[0,1]\right) .
$$

Hence the concatenation of $\boldsymbol{\omega}$ and $H_{\boldsymbol{\omega}, \tau}$ is

$$
\widetilde{R}_{\omega, \tau}=\omega H_{\omega, \tau} .
$$

To shorten the notation for an $\boldsymbol{\omega} \in \Sigma_{\ell}$ we define

$$
D[\boldsymbol{\omega}]:=\left\{\boldsymbol{\tau} \in \Sigma_{\ell}, \omega_{1} \neq \tau_{1}\right\} .
$$

Using (55) we can write

$$
V_{\varepsilon, n} \subset\left(\Pi_{\mathcal{H}}^{-1}\left(\operatorname{Bad}_{\ell, \delta}^{2}\right)\right) \bigcup \bigcup_{\boldsymbol{\omega} \in \Sigma_{\ell}} \bigcup_{\boldsymbol{\tau} \in D[\boldsymbol{\omega}]} \widetilde{R}_{\boldsymbol{\omega}, \boldsymbol{\tau}}
$$

Hence, by (50) and (7) we get

$$
\begin{aligned}
\mu\left(V_{\varepsilon, n}\right) & =K_{6}(N c)^{-\ell \delta(q-1)}+\sum_{\boldsymbol{\omega} \in \Sigma_{\ell}} \mu\left(\bigcup_{\boldsymbol{\tau} \in D[\boldsymbol{\omega}]} \boldsymbol{\omega} H_{\boldsymbol{\omega}, \boldsymbol{\tau}}\right) \\
& =K_{6}(N c)^{-\ell \delta(q-1)}+\frac{1}{N^{\ell}} \sum_{\boldsymbol{\omega} \in \Sigma_{\ell}} \mu\left(\bigcup_{\boldsymbol{\tau} \in D[\boldsymbol{\omega}]} H_{\boldsymbol{\omega}, \boldsymbol{\tau}}\right)
\end{aligned}
$$

To estimate $\mu\left(\bigcup_{\boldsymbol{\tau} \in D[\boldsymbol{\omega}]} H_{\boldsymbol{\omega}, \boldsymbol{\tau}}\right)$ we present

$$
\bigcup_{\boldsymbol{\tau} \in D[\boldsymbol{\omega}]} H_{\boldsymbol{\omega}, \boldsymbol{\tau}}=\Pi^{-1}\left(\left(\operatorname{Bad}_{\ell, \delta}^{1} \cup \bigcup_{\boldsymbol{\tau} \in D[\boldsymbol{\omega}]} \widetilde{J}_{\boldsymbol{\omega}, \boldsymbol{\tau}}\right) \times[0,1]\right),
$$


where

$$
\widetilde{J}_{\boldsymbol{\omega}, \boldsymbol{\tau}}:=J_{\boldsymbol{\omega}, \boldsymbol{\tau}} \backslash\left(h_{\boldsymbol{\omega}}^{-1}\left(\operatorname{Bad}_{\ell, \delta}^{2} \cup \operatorname{Bad}_{\ell, \delta}^{1}\right)\right)
$$

It follows from the definition of the set $\operatorname{Bad}_{\ell, \delta}^{1}$ that

$$
\text { if } t \in \widetilde{J}_{\boldsymbol{\omega}, \tau} \text { then } \varphi(t)<(N c)^{\ell \delta} \text {. }
$$

On the other hand, it follows from the definition of $\operatorname{Bad}_{\ell, \delta}^{2}$ that

$$
\sum_{\tau \in D[\omega]} \mathbb{1}_{\widetilde{J}_{\boldsymbol{\omega}, \tau}}(t) \leq(N c)^{(1+\delta) \ell}, \quad \forall t .
$$

Putting these together and using (51) and $(56)$ we get

$$
\begin{aligned}
\mu\left(\bigcup_{\boldsymbol{\tau} \in D[\boldsymbol{\omega}]} H_{\boldsymbol{\omega}, \boldsymbol{\tau}}\right) & \leq \nu_{x}\left(\operatorname{Bad}_{\ell, \delta}^{1}\right)+\nu_{x}\left(\bigcup_{\boldsymbol{\tau} \in D[\omega]} \widetilde{J}_{\boldsymbol{\omega}, \boldsymbol{\tau}}\right) \\
& \leq \nu_{x}\left(\operatorname{Bad}_{\ell, \delta}^{1}\right)+\int \sum_{\tau \in D[\omega]} \mathbb{1}_{\widetilde{J}_{\omega, \tau}}(t) \varphi(t) d t \\
& \leq M \cdot(N c)^{-\ell \delta(q-1)}+(N c)^{(1+\delta) \ell}(N c)^{\ell \delta} 3 K_{3}\left(\frac{b}{c}\right)^{\ell} \\
& \leq K_{7}\left((N c)^{-\ell \delta(q-1)}+\left(N b(N c)^{2 \delta}\right)^{\ell}\right)
\end{aligned}
$$

Putting this and (60) together we obtain that

$$
\mu\left(V_{\varepsilon, n}\right)<\left(K_{6}+K_{7}\right) r^{n},
$$

where

$$
r:=\max \left\{(N c)^{-\delta(q-1)}, N b(N c)^{2 \delta}\right\}^{\frac{\varepsilon}{-\log b}}
$$

Corollary 4.8. Let $R_{1}:=\{\mathbf{i}: \exists \mathbf{j} \neq \mathbf{i}, \Pi(\mathbf{i})=\Pi(\mathbf{j})\}$ Then $\mu\left(R_{1}\right)=0$.

Proof. It follows from Lemma 4.7 that

$$
\mu(\mathbf{i}: L(\mathbf{i})=0)=0 .
$$

Clearly,

$$
\begin{aligned}
R_{1} & =\bigcup_{n=0}^{\infty}\{\mathbf{i}: \exists \mathbf{j}, \quad|\mathbf{i} \wedge \mathbf{j}|=n, \Pi(\mathbf{i})=\Pi(\mathbf{j}) \cdot\} \\
& =\bigcup_{n=0}^{\infty} \sigma^{-n}\left\{\mathbf{i}: \exists \mathbf{j}, \quad i_{1} \neq j_{1}, \Pi(\mathbf{i})=\Pi(\mathbf{j}) \cdot\right\} \\
& =\bigcup_{n=0}^{\infty} \sigma^{-n}\{\mathbf{i}: L(\mathbf{i})=0\}
\end{aligned}
$$

This and (64) imply that $\mu\left(R_{1}\right)=0$.

The Proposition 4.6 is now immediate:

Proof of Proposition 4.6. By definition,

$$
\left\{\mathbf{i}: L\left(\sigma^{n} \mathbf{i}\right)<\mathrm{e}^{-\varepsilon n}\right\}=\sigma^{-n}\left(V_{\varepsilon, n}\right) .
$$


From this and Lemma 4.7 we get that

$$
\sum_{n} \mu\left(\left\{\mathbf{i}: L\left(\sigma^{n} \mathbf{i}\right)<\mathrm{e}^{-\varepsilon n}\right\}\right)<\infty .
$$

Let $R_{2}:=\left\{\mathbf{i}: \exists N_{0}, \forall n>N_{0} \quad L\left(\sigma^{n} \mathbf{i}\right) \geq \mathrm{e}^{-\varepsilon n}\right\}$. Then $\mu\left(R_{2}\right)=1$. It is immediate to see that every $\mathbf{i} \in R_{2} \backslash R_{1}$ is $\varepsilon$-good. This means that the set $\varepsilon$-good $\mathbf{i}$ form a set of full measure. By taking a countable intersection we get that $\mu(G)=1$.

4.2. $L^{q} \forall q$ density case. In this section we will give the proof of Theorem 4.2 . In this subsection we assume that $\nu_{x}$ is absolute continuous with $L^{q}$ density for all $q>1$, we also assume the transversality condition.

In the previous subsection we proved Lemma 4.7. In the calculation of $\mu\left(V_{\varepsilon, \ell}\right)$ we were not able to make use of the following fact: if the intervals $\operatorname{proj}_{x} S_{\boldsymbol{\omega}}\left([0,1]^{2}\right)$ and $\operatorname{proj}_{x} S_{\boldsymbol{\tau}}\left([0,1]^{2}\right)$ intersect for some $\boldsymbol{\omega}, \boldsymbol{\tau} \in \Sigma_{\ell}$, it does not necessarily mean that the parallelograms $S_{\boldsymbol{\omega}}\left([0,1]^{2}\right)$ and $S_{\boldsymbol{\tau}}\left([0,1]^{2}\right)$ intersect as well. Under the $L^{q} \forall q$ assumption this distinction can be made.

4.2.1. Number of pairs of intersecting cylinder parallelograms. In this subsection we give an upper bound on the number of intersecting (or close-by) level $\ell$ cylinders with distinct first coordinates. To state the lemma we need some preparation.

We write

$$
\Sigma_{\ell}^{2, \text { diff }}:=\left\{(\boldsymbol{\omega}, \boldsymbol{\tau}) \in \Sigma_{\ell} \times \Sigma_{\ell}: \omega_{1} \neq \tau_{1}\right\} .
$$

For an $\boldsymbol{\omega} \in \Sigma_{\ell}$ and $L>0$ let

$$
P_{\boldsymbol{\omega}}^{L}:=U_{y}\left(S_{\boldsymbol{\omega}}\left([0,1]^{2}\right), L b^{\ell}\right) \text { and } I_{\boldsymbol{\omega}}:=\operatorname{proj}_{x} S_{\boldsymbol{\omega}}\left([0,1]^{2}\right)=\operatorname{proj}_{x} P_{\boldsymbol{\omega}}
$$

Further, for $\omega, \tau \in \Sigma_{\ell}^{2, \text { diff }}$ we write

$$
P_{\boldsymbol{\omega}, \boldsymbol{\tau}}^{L}:=P_{\boldsymbol{\omega}}^{L} \cap P_{\boldsymbol{\tau}}^{L} \supset R_{\boldsymbol{\omega}, \boldsymbol{\tau}},
$$

where $R_{\omega, \tau}$ was defined in $(53)$ and

$$
I_{\boldsymbol{\omega}, \boldsymbol{\tau}}^{L}:=\operatorname{proj}_{x} P_{\omega, \tau}^{L} .
$$

Note that by transversality

$$
\left|I_{\boldsymbol{\omega}, \boldsymbol{\tau}}^{L}\right|<(L+2) K_{3} \cdot b^{\ell}
$$

Let

$$
B_{\ell}^{L}:=\#\left\{(\boldsymbol{\omega}, \boldsymbol{\tau}) \in \Sigma_{\ell}^{2, \mathrm{diff}}: P_{\boldsymbol{\omega}, \boldsymbol{\tau}}^{L} \neq \emptyset\right\}
$$

Lemma 4.9. Assume that Conditions (B1), (B3) and (B4) hold. Then for every $L>0$

$$
\limsup _{n \rightarrow \infty} \frac{1}{n} \log B_{n}^{L} \leq \log \left(N^{2} c^{2}\right) \text {. }
$$

Proof. Choose some $\widetilde{n}>1$. Let $n \geq \widetilde{n}$. Let us define a finite sequence $n_{i}$ as follows: $n_{0}:=n$ and

$$
n_{k}:=\left\lfloor\left(\frac{\log c}{\log b}\right)^{k} n\right\rfloor .
$$

Let $\mathcal{K}:=\max \left\{k \geq 0: n_{k} \geq \tilde{n}\right\}$. Naturally,

$$
B_{n_{\mathcal{K}}}^{L} \leq N^{2 n_{\mathcal{K}}}
$$

We show that for every $q>1$ there exists $K=K(q, L)$ such that for every $0 \leq k \leq \mathcal{K}$

$$
B_{n_{k}}^{L} \leq B_{n_{k+1}}^{L} \cdot\left(K(N c)^{n_{k}-n_{k+1}} c^{-\left(n_{k}-n_{k+1}\right) / q}\right)^{2} .
$$


Let $\boldsymbol{\omega}, \boldsymbol{\tau} \in \Sigma_{n_{k}}$. Assume that

$$
P_{\omega}^{L} \cap P_{\tau}^{L} \neq \emptyset .
$$

Observe that this is possible only if both

$$
\begin{aligned}
& \text { (a): } P_{\left.\boldsymbol{\omega}\right|_{n_{k+1}} ^{L}}^{L} \cap P_{\left.\boldsymbol{\tau}\right|_{n_{k+1}}}^{L} \neq \emptyset \text { and } \\
& \text { (b): } I_{\boldsymbol{\omega}} \cap I_{\left.\boldsymbol{\omega}\right|_{n_{k+1}},\left.\boldsymbol{\tau}\right|_{n_{k+1}}}^{L} \neq \emptyset \text { and } I_{\boldsymbol{\tau}} \cap I_{\left.\boldsymbol{\omega}\right|_{n_{k+1}},\left.\boldsymbol{\tau}\right|_{n_{k+1}}}^{L} \neq \emptyset
\end{aligned}
$$

hold. By (69) and (71)

$$
\left|I_{\left.\boldsymbol{\omega}\right|_{k+1},\left.\boldsymbol{\tau}\right|_{n_{k+1}}}\right| \leq(L+2) K_{3} b^{n_{k+1}} \approx c^{n_{k}}
$$

Hence by Corollary 7.6 , for every $\left.\boldsymbol{\omega}\right|_{n_{k+1}},\left.\boldsymbol{\tau}\right|_{n_{k+1}}$ there are at most $C(L, q)(N c)^{n_{k}-n_{k+1}} c^{-\left(n_{k}-n_{k+1}\right) / q}$ words $\boldsymbol{\omega} \in \Sigma_{n_{k}}$ such that $I_{\boldsymbol{\omega}} \cap I_{\left.\boldsymbol{\omega}\right|_{n_{k+1}},\left.\boldsymbol{\tau}\right|_{n_{k+1}}}^{L} \neq \emptyset$. In the same way there are at most $C(L, q)(N c)^{n_{k}-n_{k+1}} c^{-\left(n_{k}-n_{k+1}\right) / q}$ words $\boldsymbol{\tau} \in \Sigma_{n_{k}}$ such that $I_{\boldsymbol{\tau}} \cap I_{\left.\boldsymbol{\omega}\right|_{n_{k+1}},\left.\boldsymbol{\tau}\right|_{n_{k+1}}}^{L} \neq \emptyset$. So, taking into consideration condition (a) above, we obtain (72).

Thus, by induction

$$
B_{n_{0}}^{L} \leq(K)^{2 \mathcal{K}} N^{2 n_{\mathcal{K}}}(N c)^{2\left(n_{0}-n_{\mathcal{K}}\right)} c^{-2\left(n_{0}-n_{\mathcal{K}}\right) / q} .
$$

But by definition of the sequence $\left\{n_{k}\right\}, n_{0}=n, n_{\mathcal{K}} \approx \widetilde{n}$, and there exist constants $c_{1}, c_{2} \in \mathbb{R}$ such that $\mathcal{K} \leq c_{1} \log n+c_{2}$. Therefore,

$$
B_{n}^{L} \leq C(q, L) \cdot K(q, L)^{2 c_{1} \log n} \cdot c^{-n / q} \cdot(N c)^{2 n},
$$

and passing with $q$ to infinity proves the assertion.

4.2.2. The corellation dimension. First we recall the definition of the correlation dimension (see [17]).

Definition 4.10. Let $\mathrm{m}$ be a positive bounded regular Borel measure on $\mathbb{R}^{d}$ with bounded support $\operatorname{spt}(\mathfrak{m})$. For every $r>0$ let $\left\{B_{i}^{(r)}\right\}_{i}$ be the r-mesh cubes that intersect $\operatorname{spt}(\mathfrak{m})$. For a $q>0, q \neq 1$ we define

$$
\tau(q):=\liminf _{r \rightarrow 0^{+}} \frac{\log \sum_{i} \mathfrak{m}\left(B_{i}^{(r)}\right)^{q}}{\log r} .
$$

Equivalently we could define $\tau(q)$ (see [17]) in either of the following two ways: let

$$
I_{r}(q):=\int_{\mathbb{R}^{d}} \mathfrak{m}(B(x, r))^{q} d x \text { and } \widetilde{I}_{r}(q):=\int_{\mathbb{R}^{d}} \mathfrak{m}(B(x, r))^{q-1} d \mathfrak{m}(x) .
$$

Then

$$
\tau(q)=\liminf _{r \rightarrow 0^{+}} \frac{\log I_{r}(q)}{\log r}-d=\liminf _{r \rightarrow 0^{+}} \frac{\log \widetilde{I}_{r}(q)}{\log r} .
$$

For $q>1$ the $L^{q}$-dimension of $\mathfrak{m}$ is defined as

$$
\underline{\operatorname{dim}}_{\mathrm{q}}(\mathfrak{m}):=\frac{\tau(q)}{q-1} .
$$


It was proved by Hunt and Kaloshin [15, Proposition 2.1] that

$$
\begin{aligned}
\underline{\operatorname{dim}}_{\mathrm{q}}(\mathfrak{m}) & =\sup \left\{t \geq 0: \int\left(\int \frac{d \mathfrak{m}(y)}{|x-y|^{t}}\right)^{q-1} d \mathfrak{m}(x)<\infty\right\} \\
& =\inf \left\{t \geq 0: \int\left(\int \frac{d \mathfrak{m}(y)}{|x-y|^{t}}\right)^{q-1} d \mathfrak{m}(x)=\infty\right\} \\
& =\sup \left\{s: \int_{0}^{\infty} r^{-s(q-1)-1} \widetilde{I}_{r}(q) d r<\infty\right\} .
\end{aligned}
$$

If we apply this for $q=2$ we get the correlation dimension of the measure $\mathfrak{m}$

$$
\operatorname{dim}_{C}(\mathfrak{m}):=\underline{\operatorname{dim}}_{2}(\mathfrak{m}) .
$$

It follows from $(78)$ that

$$
\operatorname{dim}_{\mathrm{C}}(\mathfrak{m}) \leq \operatorname{dim}_{\mathrm{H}}(\mathfrak{m})
$$

Proof of Theorem 4.2. We will prove that under Assumption B

$$
\operatorname{dim}_{C} \nu \geq \min \left(2,1+\frac{\log (N c)}{-\log b}\right) .
$$

Let $\ell>0$ and consider the grid of size $r=2 b^{\ell}$. Let $B=\left[x-b^{\ell}, x+b^{\ell}\right] \times\left[y-b^{\ell}, y+b^{\ell}\right]$ be one of the $r$-mesh cubes. Let $|\boldsymbol{\tau}|=\ell$ be such that $B$ intersects $S_{\boldsymbol{\tau}}\left([0,1]^{2}\right)$. Then $S_{\boldsymbol{\tau}}^{-1}\left(B \cap S_{\boldsymbol{\tau}}\left([0,1]^{2}\right)\right)$ is contained in some vertical strip of width $b^{\ell} / c^{\ell}$, hence by Corollary 7.6

$$
\nu\left(S_{\boldsymbol{\tau}}^{-1}\left(B \cap S_{\boldsymbol{\tau}}\left([0,1]^{2}\right)\right)\right) \leq C(q)(b / c)^{\ell(1-1 / q)} .
$$

Let $L:=(c-b)^{-1} \cdot \max _{i \leq m}\left|d_{i}\right|$ be an upper bound on the maximal slope of (the principal axis of) our cylinders. Then if the cylinder $S_{\boldsymbol{\tau}}\left([0,1]^{2}\right),|\boldsymbol{\tau}|=\ell$ intersects $B$, it must also intersect at least one of vertical intervals: either $\left\{x-b^{\ell}\right\} \times\left[y-(2 L+1) b^{\ell}, y+(2 L+1) b^{\ell}\right]$ or $\left\{x+b^{\ell}\right\} \times\left[y-(2 L+1) b^{\ell}, y+(2 L+1) b^{\ell}\right]$. Let us denote the number of cylinders $S_{\tau}\left([0,1]^{2}\right)$ intersecting $\left\{x-b^{\ell}\right\} \times\left[y-(L+1) b^{\ell}, y+(L+1) b^{\ell}\right]$ by $Z_{1}(x, y)$ and the number of cylinders intersecting $\left\{x+b^{\ell}\right\} \times\left[y-(L+1) b^{\ell}, y+(L+1) b^{\ell}\right]$ by $Z_{2}(x, y)$. Using a similar estimate as in the proof of Lemma 4.7 , in equation 60 we can write

$$
\nu(B) \leq C(q) N^{-\ell} \cdot(b / c)^{\ell(1-1 / q)} \cdot\left(Z_{1}(x, y)+Z_{2}(x, y)\right)
$$

hence

$$
(\nu(B))^{2} \leq C(q)^{2} N^{-2 \ell} \cdot(b / c)^{2 \ell(1-1 / q)} \cdot\left(2 Z_{1}^{2}(x, y)+2 Z_{2}^{2}(x, y)\right)
$$

Thus,

$$
\sum \nu\left(B_{i}^{(r)}\right)^{2} \leq C(q)^{2} N^{-2 \ell}(b / c)^{2 \ell(1-1 / q)} \cdot\left(\sum Z_{1}^{2}\left(x_{i}, y_{i}\right)+\sum Z_{2}^{2}\left(x_{i}, y_{i}\right)\right)
$$

where $B_{i}^{(r)}$ is the $i$-th $r$-mesh cube. It is enough to estimate the first sum, the second is analogous. The interval $\left\{x-b^{\ell}\right\} \times\left[y-(2 L+1) b^{\ell}, y+(2 L+1) b^{\ell}\right]$ intersects $S_{\tau}\left([0,1]^{2}\right)$ if and only if the point $\left(x-b^{\ell}, y\right)$ is contained in $S_{\tau}([0,1] \times[-2 L-1,2 L+2])$. Hence, $Z_{1}^{2}(x, y)$ is equal to the number of pairs $\boldsymbol{\omega}, \boldsymbol{\tau} \in \Sigma_{\ell} \times \Sigma_{\ell}$ such that $P_{\boldsymbol{\omega}, \tau}^{2 L+1}$ contains $\left(x-b^{\ell}, y\right)$.

For every $\boldsymbol{\omega}, \boldsymbol{\tau} \in \Sigma_{\ell}$ we write $k:=|\boldsymbol{\omega} \wedge \boldsymbol{\tau}|$. Then for $\boldsymbol{\alpha}:=\boldsymbol{\omega} \wedge \boldsymbol{\tau} \in \Sigma_{k}$, there exists $\beta, \gamma \in \Sigma_{\ell-k}$ such that

$$
\omega=\alpha \beta \text { and } \boldsymbol{\tau}=\alpha \gamma
$$




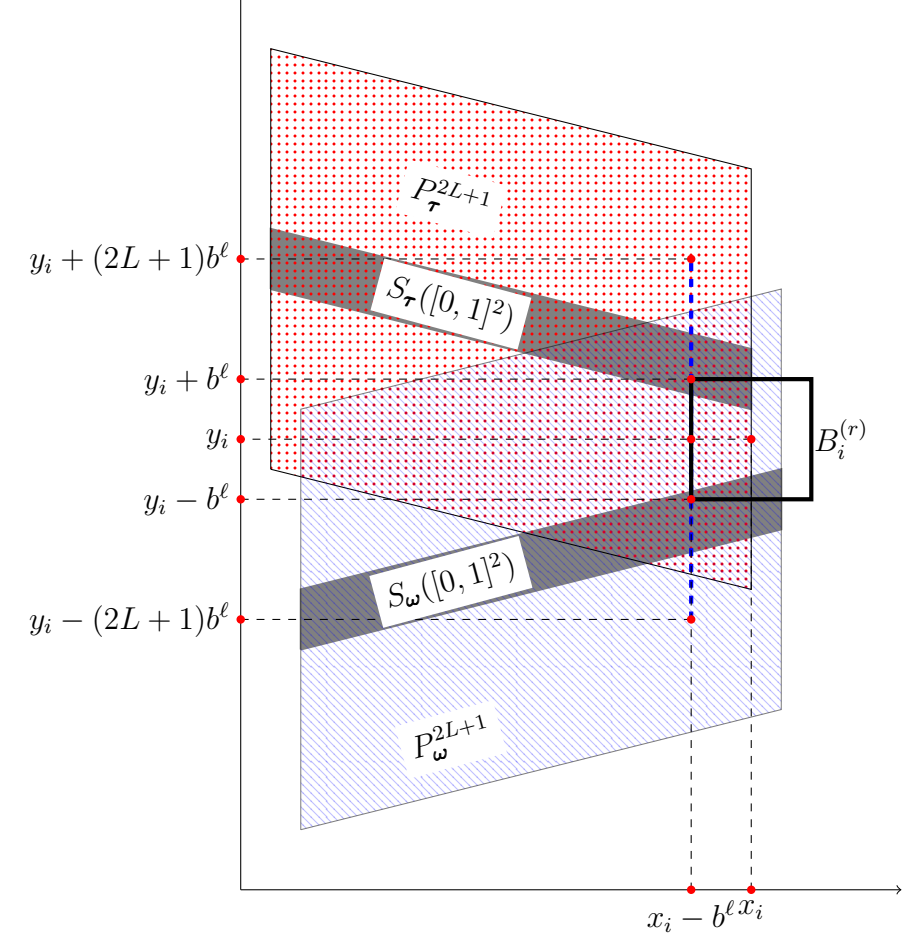

FiguRE 2. The region where (D4) holds.

With this notation we present this sum as

$$
\sum Z_{1}^{2}\left(x_{i}, y_{i}\right)=\sum_{k=0}^{\ell} \sum_{\boldsymbol{\alpha} \in \Sigma_{k}} \sum_{(\boldsymbol{\beta}, \boldsymbol{\gamma}) \in \Sigma_{\ell-k}^{2, \text { diff }}}\left|\left\{i ;\left(x_{i}-b^{\ell}, y_{i}\right) \in P_{\boldsymbol{\omega}, \boldsymbol{\tau}}^{2 L+1}\right\}\right| .
$$

Given $\boldsymbol{\alpha}$, the last sum can be estimated by

$$
\sum_{(\boldsymbol{\beta}, \boldsymbol{\gamma}) \in \Sigma_{\ell-k}^{2, \text { diff }}}\left|\left\{i ;\left(x_{i}-b^{\ell}, y_{i}\right) \in P_{\boldsymbol{\omega}, \boldsymbol{\tau}}^{2 L+1}\right\}\right| \leq C B_{\ell-k}^{2 L+1}\left(\frac{c}{b}\right)^{k} .
$$

Namely, by the Transversality Condition we can apply (69) which yields that $\left|I_{\boldsymbol{\beta}, \boldsymbol{\gamma}}^{2 L+1}\right| \leq(2 L+5) K_{3} b^{\ell-k}$ and $\left|I_{\boldsymbol{\omega}, \boldsymbol{\tau}}^{2 L+1}\right|=c^{k} \cdot\left|I_{\boldsymbol{\beta}, \boldsymbol{\gamma}}^{2 L+1}\right|$. Thus we have only $C \cdot(c / b)^{k}$ different $\left(x_{i}-b^{\ell}\right)^{\prime}$ s in $I_{\boldsymbol{\omega}, \boldsymbol{\tau}}^{2 L+1}$ and for each of them we have at most $2 L+2$ different $y_{i}{ }^{\prime}$ in $P_{\boldsymbol{\omega}, \boldsymbol{\tau}}^{L+1}$. Then (80) follows from 70 .

Applying Lemma 4.9 and noting that $\boldsymbol{\alpha}$ can take $N^{k}$ values, we get

$$
\sum Z_{1}^{2}\left(x_{i}, y_{i}\right) \leq C N^{2 \ell} c^{2 \ell} o\left(N^{\ell \varepsilon}\right) \cdot \sum_{k=0}^{\ell}(N c b)^{-k}
$$

The last sum is a geometric series, hence it is bounded by a constant when $N c b>1$ and by $(N c b)^{-\ell}$ when $N c b<1$. When $N c b=1$ this sum equals $\ell+1=o\left(N^{\ell \varepsilon}\right)$. Thus,

$$
\sum \nu\left(B_{i}^{(r)}\right)^{2} \leq C b^{\ell \cdot \min (2,1+\log (N c) /-\log b)}(b / c)^{-2 \ell / q} O\left(N^{2 \ell \varepsilon}\right) .
$$

Passing with $q$ to infinity and $\varepsilon$ to 0 we get

$$
\tau(2) \geq \min \left(2,1+\frac{\log (N c)}{-\log b}\right) .
$$




\section{DiRECTIOn-y DOMinates}

Finally, in this section we turn to the case when the direction-y dominates. In this direction, we have only a mild development on the way of understanding the overlapping self-affine systems. The result can be considered as an extension of [6, Theorem B] and [3, Theorem 4.8, Theorem 4.11].

Similarly to the Section 1.5, we define the backward Furstenberg-Kifer measure and IFS. This measure is supported on the directions, associated to the strong-stable directions. We note that in the case, when direction- $x$ dominates, the backward Furstenberg-Kifer measure is supported on the singleton $\{(0,1)\}$.

Consider again the vertical line $\xi:=\left\{(1, z) \in \mathbb{R}^{2}: z \in \mathbb{R}\right\}$ on the plane and identify $(1, z) \in \xi$ with $\widetilde{z} \in \mathbb{R}$. Moreover, let $\mathcal{B}$ be the self-similar IFS on $\xi$ defined by

$$
\mathcal{B}:=\left\{g_{i}(\widetilde{z}):=\frac{c}{b} \widetilde{z}-\frac{d_{i}}{b}\right\}_{i=1}^{N}
$$

Let us define the natural projection by $\Pi_{B F}: \Sigma \rightarrow \xi$ in the usual way:

$$
\Pi_{B F}(\mathbf{i}):=-\frac{d_{i_{1}}}{b}-\sum_{k=2}^{\infty} \frac{d_{i_{k}}}{b} \cdot\left(\frac{c}{b}\right)^{k-1}
$$

Similarly, to 12$)$, we have that the action of $\left\{T_{i}^{-1}\right\}_{i=1}^{N}$ on the projective line is described by the maps $\widehat{T}_{i}: \xi \rightarrow \xi$

$$
\widehat{T}_{i}(\widetilde{z}):=c \cdot T_{i}^{-1} \cdot\left(\begin{array}{c}
1 \\
z
\end{array}\right)
$$

where $\widetilde{z} \in \xi$ is $\widetilde{z}=(1, z)$.

Assumption C. We assume that

(C1) $c<\frac{1}{N}$

(C2) $b>c$

(C3) The backward Furstenberg-Kifer IFS $\mathcal{B}$ satisfies Hochman's exponential separation condition,

(C4) H satisfies Hochman's exponential separation condition,

(C5) $\frac{\log N}{\log (b / c)} \geq \min \left\{1, \frac{\log N}{-\log b}, 2\left(1-\frac{\log N}{-\log c}\right)\right\}$. 


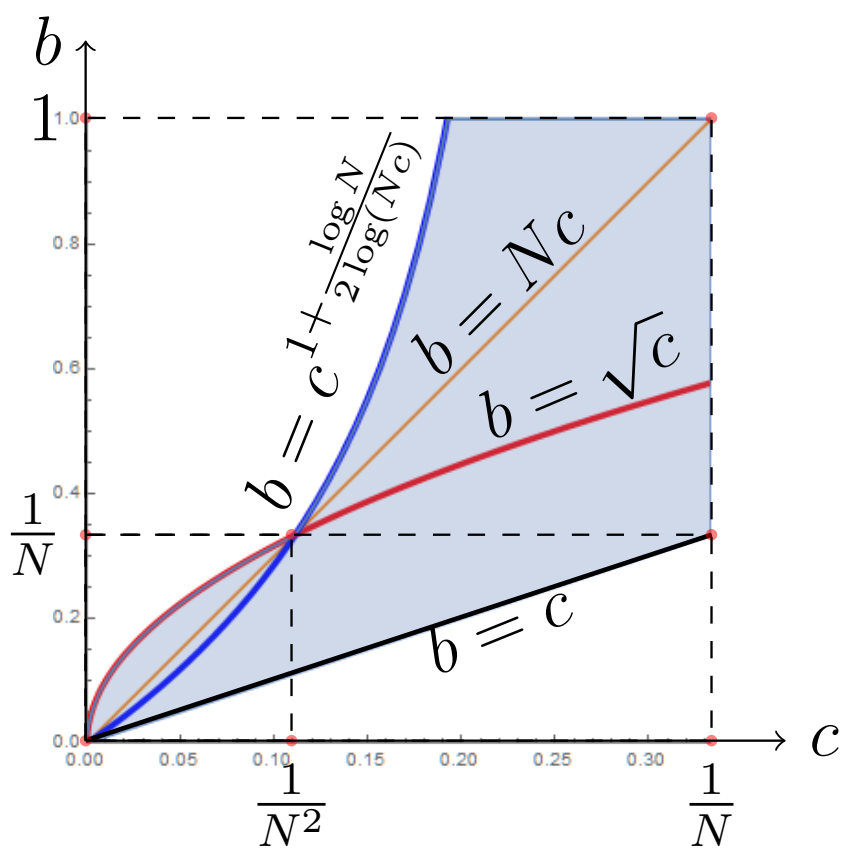

FiguRE 3. The region where (C5) holds.

Conditions ( $\mathrm{C} 1)$ and $(\mathrm{C} 4)$ are devoted to be able to handle the projection entropy (defined in Section 1.7.2). Condition (C3) allows us to calculate the dimension of the backward Furstenberg-Kifer measure and condition (C5) ensures that its dimension is larger than some possible exceptional set of orthogonal projections, for which the dimension drops.

Theorem 5.1. Let $\mathcal{S}$ be a self-affine IFS of the form (2) satisfying Assumption $C$. Then

$$
\operatorname{dim}_{\mathrm{H}}(\Lambda)=\operatorname{dim}_{\mathrm{H}}(\nu)=\min \left\{\frac{\log N}{-\log b}, 1+\frac{\log (N b)}{-\log c}\right\} .
$$

As previously, let $\mu$ be the uniform Bernoulli measure on the symbolic space and let $\nu$ be its projection by the mapping $\Pi$, defined in Section 1.4 .

For a $\theta$ proper subspace of $\mathbb{R}^{2}$, let us denote the orthogonal projection from $\mathbb{R}^{2}$ to the subspace $\theta^{\perp}$ (orthogonal subspace to $\theta$ ) by $\operatorname{Proj}_{\theta}$. By [4, Theorem 2.2],

$$
\operatorname{dim} \nu=\frac{h_{\Pi}(\mu)}{-\log c}+\left(1-\frac{-\log b}{-\log c}\right) \operatorname{dim}\left(\operatorname{Proj}_{\theta}\right)_{*} \nu \text { for }\left(\Pi_{B F}\right)_{*} \mu \text {-a.e. } \theta,
$$

where $h_{\Pi}(\mu)$ is the projection entropy, defined in Section 1.7.2.

Lemma 5.2. If (C1), (C2) and (C4) in Assumption C hold then $h_{\Pi}(\mu)=h(\mu)=\log N$.

Proof. Let us define a lifted IFS on $[0,1]^{3}$ and a derived IFS on $\{0\} \times[0,1]^{2}$, as follows

$$
\begin{gathered}
\widehat{\Phi}:=\left\{\widehat{S}_{i}(x, y, z)=\left(c x+u_{i}, b y+d_{i} x+v_{i}, \rho z+w_{i}\right)\right\}_{i=1}^{m} \text { and } \\
\widetilde{\Phi}:=\left\{\widetilde{S}_{i}(y, z)=\left(c x+u_{i}, \rho z+w_{i}\right)\right\}_{i=1}^{m},
\end{gathered}
$$

where $0<\rho<\min \{|c|,|b|\}$ and $w_{i} \in \mathbb{R}$ are chosen such that

$$
\widehat{S}_{i}\left([0,1]^{3}\right) \cap \widehat{S}_{j}\left([0,1]^{3}\right)=\emptyset \text { and } \widetilde{S}_{i}\left([0,1]^{2}\right) \cap \widetilde{S}_{j}\left([0,1]^{2}\right)=\emptyset \text { for every } i \neq j .
$$

Denote the natural projections of $\widehat{\Phi}$ and $\widetilde{\Phi}$ by $\widehat{\Pi}$ and $\widetilde{\Pi}$ respectively. Let us define $\widehat{\nu}=\widehat{\Pi}_{*} \mu$ and $\widetilde{\nu}=\widetilde{\Pi}_{*} \mu$ the push-down measures. 
We note that the Lyapunov exponents coincide for every measure $\widehat{\nu}, \widetilde{\nu}$, and $\nu$ for the appropriate directions. Applying [4, Corollary 2.9] and [11, Theorem 2.11], we have

$$
\begin{aligned}
\operatorname{dim}_{H} \widehat{\nu}= & \frac{h(\mu)}{-\log \rho}+\left(\frac{-\log \rho+\log c}{-\log \rho}\right) \operatorname{dim} \nu+ \\
& \left(\frac{-\log c+\log b}{-\log \rho}\right) \operatorname{dim}\left(\operatorname{Proj}_{\theta}\right)_{*} \nu \text { for }\left(\Pi_{B F}\right)_{*} \mu \text {-a.e. } \theta, \\
\operatorname{dim}_{H} \widetilde{\nu}= & \frac{h_{\Pi_{\mathcal{H}}}(\mu)}{-\log c}+\frac{h(\mu)-h_{\Pi_{\mathcal{H}}}(\mu)}{-\log \rho} .
\end{aligned}
$$

By (84) and 86 we have

$$
\operatorname{dim}_{H} \widehat{\nu}=\frac{h(\mu)-h_{\Pi}(\mu)}{-\log \rho}+\operatorname{dim} \nu .
$$

Let us introduce measurable partitions of $[0,1]^{3}$ by $\xi(a, y):=\{a\} \times\{y\} \times[0,1]$ and $\tau(a):=\{a\} \times[0,1] \times[0,1]$. Moreover, define a measurable partition of $[0,1] \times\{0\} \times[0,1]$ by $\zeta(a)=\{a\} \times\{0\} \times[0,1]$ and a measurable partition of $[0,1]^{2} \times\{0\}$ by $\eta(a)=\{a\} \times$ $[0,1] \times\{0\}$.

By Rokhlin's Theorem there are families of conditional measures $\widehat{\nu}_{a, y}^{\xi}, \widehat{\nu}_{a}^{\tau}, \widetilde{\nu}_{a}^{\zeta}$ and $\nu_{a}^{\eta}$ on the partitions respectively, uniquely defined up to zero measure sets.

By definition of conditional measures and the partition $\tau, \widehat{\nu}=\int \widehat{\nu}_{a}^{\tau} d \nu_{x}(a)$, where $\nu_{x}=$ $\left(\Pi_{\mathcal{H}}\right)_{*} \mu$, see (29). On the other hand, $\widehat{\nu}=\int \widehat{\nu}_{a, y}^{\xi} d \nu(a, y)=\iint \widehat{\nu}_{a, y}^{\xi} d \nu_{a}^{\eta}(y) d \nu_{x}(a)$. Thus,

$$
\widehat{\nu}_{a}^{\tau}=\int \widehat{\nu}_{a, y}^{\xi} d \nu_{a}^{\eta}(y) \text { for } \nu_{x^{-}} \text {a.e. } a .
$$

Let proj: $[0,1]^{3} \mapsto\{0\} \times[0,1]^{2}$ be the orthogonal projection to the $y, z$-coordinate plane. Since $(\text { proj) })_{*} \widehat{\nu}_{a}^{\tau}=\widetilde{\nu}_{a}^{\zeta}$ for $\nu_{x}$-a.e. $a$, we get that

$$
\widetilde{\nu}_{a}^{\zeta}=\int(\operatorname{proj})_{*} \widehat{\nu}_{a, y}^{\xi} d \nu_{a}^{\eta}(y) \text { for } \nu_{x} \text {-a.e. } a \text {. }
$$

Applying [4, Theorem 7.1] we have

$$
\begin{aligned}
& \operatorname{dim} \widehat{\nu}_{a, y}^{\xi}=\frac{h(\mu)-h_{\Pi}(\mu)}{-\log \rho} \text { for } \nu \text {-a.e. }(a, y) \\
& \operatorname{dim} \widetilde{\nu}_{a}^{\zeta}=\frac{h(\mu)-h_{\Pi_{\mathcal{H}}}(\mu)}{-\log \rho} \text { for } \nu \text {-a.e. } a .
\end{aligned}
$$

By assumptions (C1) and (C4), we may apply [11, Theorem 2.8] and [13, Theorem 1.1], and therefore

Therefore $\operatorname{dim} \widetilde{\nu}_{a}^{\zeta}=0$ for $\nu_{x}$-a.e. $a$.

$$
\operatorname{dim} \nu_{x}=\frac{h_{\Pi_{\mathcal{H}}}(\mu)}{-\log c}=\frac{h(\mu)}{-\log c} .
$$

By $(90)$, if $\widetilde{\nu}_{a}^{\zeta}(R)=0$ for a Borel set $R \subseteq\{a\} \times\{0\} \times[0,1]$ then $(\operatorname{proj})_{*} \widehat{\nu}_{a, y}^{\xi}(R)=0$ for $\nu_{a}^{\eta}$ a.e $y$. Thus, by the definition of the Hausdorff dimension $\operatorname{dim}_{H} \widetilde{\nu}_{a}^{\zeta} \geq \operatorname{dim}_{H}(\operatorname{proj})_{*} \widehat{\nu}_{a, y}^{\xi}=$ $\operatorname{dim}_{H} \widehat{\nu}_{a, y}^{\xi}$ for $\nu$-a.e $(a, y)$. Hence $\operatorname{dim}_{H} \widehat{\nu}_{a, y}^{\xi}=0$ for $\nu$-a.e. $(a, y)$, which implies that $h(\mu)=h_{\Pi}(\mu)$.

Lemma 5.3. Let $\mathcal{S}$ be a self-affine IFS of the form (2) satisfying the assumptions of Assumption $C$. Then

$$
\operatorname{dim} \nu \geq \min \left\{2 \frac{h(\mu)}{-\log c}, \frac{h(\mu)}{-\log b}, 1+\frac{h(\mu)+\log b}{-\log c}\right\}
$$




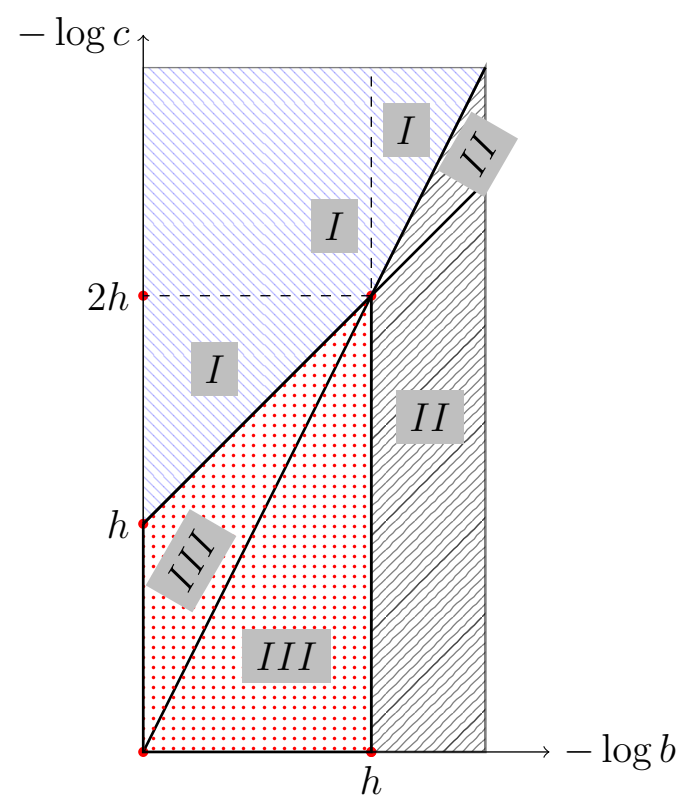

FIGURE 4. Region I,II and III corresponds to the area where the minimum in (91) is attained at the first, second or third expression respectively.

Proof. By [13, Theorem 1.1],

$$
\operatorname{dim}\left(\Pi_{B F}\right)_{*} \mu=\min \left\{1, \frac{h(\mu)}{\log b-\log c}\right\} .
$$

By [3, Lemma 4.3],

$$
\operatorname{dim}\left(\operatorname{Proj}_{\theta}\right)_{*} \nu \geq \min \left\{\operatorname{dim}\left(\Pi_{B F}\right)_{*} \mu, \operatorname{dim} \nu\right\} \text { for }\left(\Pi_{B F}\right)_{*} \mu \text {-a.e. } \theta \text {. }
$$

Let us define a sequence $\left\{x_{n}\right\}_{n=0}^{\infty}$ inductively as follows. Let $x_{0}=\frac{h(\mu)}{-\log c}$ and $x_{n}=r\left(x_{n-1}\right)$ for $n \geq 1$, where

$$
r(x)=\frac{h(\mu)}{-\log c}+\left(1-\frac{\log b}{\log c}\right) \min \left\{1, \frac{h(\mu)}{\log (b / c)}, x\right\} .
$$

It is easy to see that

$$
\lim _{n \rightarrow \infty} x_{n}=\min \left\{2 \frac{h(\mu)}{-\log c}, \frac{h(\mu)}{-\log b}, 1+\frac{h(\mu)+\log b}{-\log c}\right\}
$$

which is the fixed point of $x \mapsto r(x)$. By applying (84) and Lemma 5.2, one can show by induction that $\operatorname{dim} \mu \geq x_{n}$ for every $n \geq 0$, as required.

Proof of Theorem 5.1. By [19, Proposition 6.1] and (92), if

$$
\operatorname{dim}\left(\Pi_{B F}\right)_{*} \mu>\min \{\operatorname{dim} \nu, 2-\operatorname{dim} \nu\}
$$

then

$$
\operatorname{dim}\left(\operatorname{Proj}_{\theta}\right)_{*} \nu=\min \{1, \operatorname{dim} \nu\} \text { for }\left(\Pi_{B F}\right)_{*} \mu \text {-a.e. } \theta \text {. }
$$

But by [13, Theorem 1.1],

$$
\operatorname{dim}\left(\Pi_{B F}\right)_{*} \mu=\min \left\{1, \frac{h(\mu)}{\log b-\log c}\right\} .
$$


and by Lemma 5.3 ,

$$
\min \left\{2 \frac{h(\mu)}{-\log c}, \frac{h(\mu)}{-\log b}, 1+\frac{h(\mu)+\log b}{-\log c}\right\} \leq \operatorname{dim} \nu .
$$

which together with assumption (C5) implies (93). Thus, (84), Lemma 5.2 and (94) verify the statement.

\section{EXAMPLES}

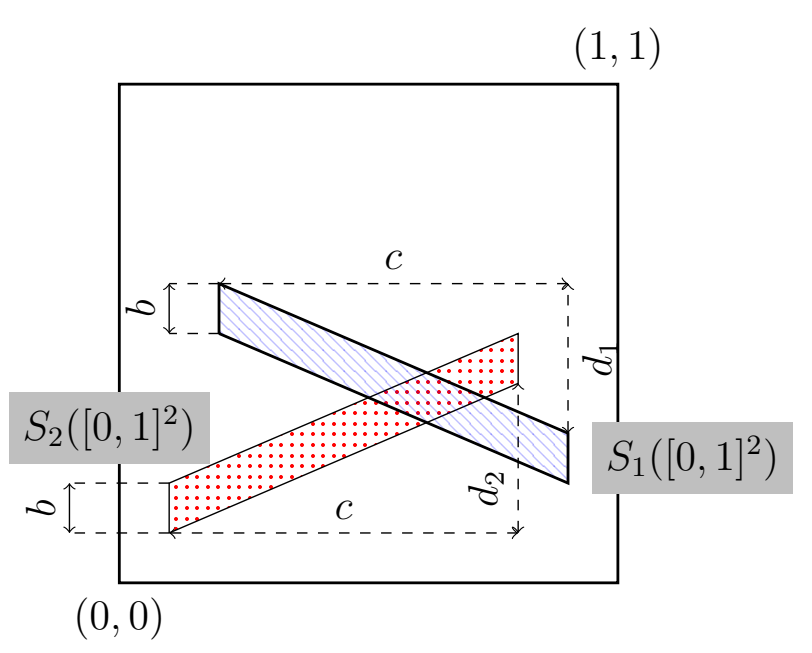

(A) In Example 6.1

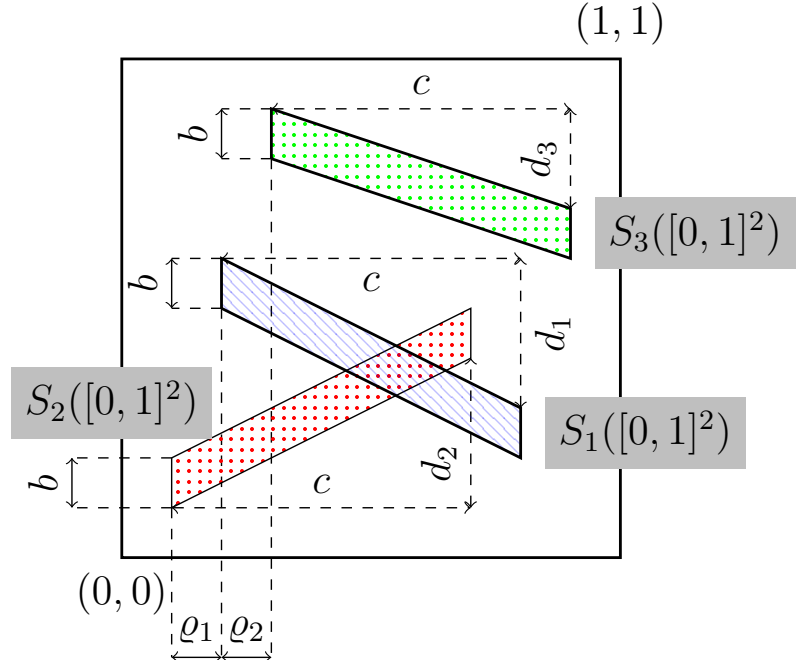

(B) and in Example 6.2 and 6.3 .

FiguRE 5. negative values of $d_{i}$ correspond to "decreasing" parallelograms. (In the Example 6.2, $\rho_{1}=\rho_{2}$.)

6.1. Examples for the direction- $x$ dominates case. We present three examples. In the first and second case we can apply Theorem 4.1 and in the third example we can apply Theorem 4.2. In all examples $c$ can be chosen as an arbitrary element of a parameter interval except a small exceptional set which is going to be $E_{1} \subset(0.5,1), E_{2} \subset(1 / 3,1)$ and $E_{3} \subset\left(\frac{1}{\sqrt{3}}, 1\right)$ in the examples respectively with the properties:

$$
\operatorname{dim}_{\mathrm{H}}\left(E_{1}\right)=\operatorname{dim}_{\mathrm{H}}\left(E_{2}\right)=0 \text { and } \mathcal{L} \text { eb }\left(E_{3}\right)=0 .
$$

The precise definition of these exceptional sets are given in Section 6.1.2.

Example 6.1. Let $\mathcal{S}$ be an IFS of the form (2), where

- $N=2$.

- We choose an arbitrary $c \in\left(\frac{1}{2}, 1\right) \backslash E_{1}$ (see (105)) and $0<b<c / 2$.

- We assume that $u_{1} \neq u_{2}, d_{1} \neq d_{2}$ and $v_{1}, v_{2}$ are selected in such a way that the IFS $\mathcal{S}:=\left\{S_{1}, S_{2}\right\}$ satisfies $S_{i}\left([0,1]^{2}\right) \subset[0,1]^{2}$. Then by Theorem 4.1 we have

$$
\operatorname{dim}_{H}(\Lambda)=1+\frac{\log (2 c)}{-\log b}
$$

(See Figure 5a.)

We can apply Theorem 4.1 in Example 6.1 because Assumption A holds. Namely, it is obvious that assumptions (A1)-(A2) hold. Assumption (A3) follows from choice of $E_{1}$ (see (105)). Assumption (A4), (the transversality condition) follows from the fact that the associated 3-dimensional IFS $\widetilde{\mathcal{S}}$, defined in (14), satisfies SSP. Namely, the third 
coordinate of $\widetilde{\mathcal{S}}$ is an IFS $\mathcal{F}$ on the line, defined in $(9)$. This consists of two maps with distinct fixed points and the sum of their contraction ratios is less than 1 . This means that the SSP holds for $\mathcal{F}$. Consequently, the SSP holds for $\widetilde{\mathcal{S}}$. Hence the transversality condition (A4) also holds.

Example 6.2. (a): $N=3$.

(b): We fix an arbitrary $c \in\left(\frac{1}{3}, 1\right) \backslash E_{2}$. (See (106).)

(c): Let $b \in\left(0, \frac{c}{2}\right) \cap\left(0, \frac{1}{3}\right)$.

(d): $u_{1}, u_{2}, u_{3}$ are pairwise different.

(e): We assume that $d_{1}<d_{2}$ and we choose $d_{3}$ from the interval

$$
d_{3} \in\left(d_{2}\left(2-\frac{c}{b}\right)+d_{1}\left(\frac{c}{b}-1\right), d_{1}\left(2-\frac{c}{b}\right)+d_{2}\left(\frac{c}{b}-1\right)\right) .
$$

Note that this holds for example if $d_{1}<d_{3}<d_{2}$.

(f): We choose the vertical translation parameters (like on the right hand side of Figure 5b) so that

$$
S_{3}\left([0,1]^{2}\right) \cap\left(S_{1}\left([0,1]^{2}\right) \cup S_{2}\left([0,1]^{2}\right)\right)=\emptyset,
$$

and $S_{i}\left([0,1]^{2}\right) \subset[0,1]^{2}$ for $i=1,2,3$.

Then we have

$$
\operatorname{dim}_{\mathrm{H}}(\Lambda)=1+\frac{\log (3 c)}{-\log b}
$$

(See Figure 5b.)

\section{Example 6.3. (a): $N=3$.}

(b): We fix an arbitrary $c \in\left(\frac{1}{\sqrt{3}}, 1\right) \backslash E_{3}$. (See 106).)

(c): Let $b \in\left(0, \frac{c}{2}\right)$.

(d): $u_{1}, u_{2}, u_{3}$ are consecutive elements of an arithmetic progression (not necessarily in this order).

(e): We assume that $d_{1}<d_{2}$ and we choose $d_{3}$ from the interval

$$
d_{3} \in\left(d_{2}\left(2-\frac{c}{b}\right)+d_{1}\left(\frac{c}{b}-1\right), d_{1}\left(2-\frac{c}{b}\right)+d_{2}\left(\frac{c}{b}-1\right)\right) .
$$

Note that this holds for example if $d_{1}<d_{3}<d_{2}$.

(f): We choose the vertical translation parameters (like on the right hand side of Figure 5b) so that

$$
S_{3}\left([0,1]^{2}\right) \cap\left(S_{1}\left([0,1]^{2}\right) \cup S_{2}\left([0,1]^{2}\right)\right)=\emptyset,
$$

and $S_{i}\left([0,1]^{2}\right) \subset[0,1]^{2}$ for $i=1,2,3$.

Then we have

$$
\operatorname{dim}_{H}(\Lambda)=\min \left\{2,1+\frac{\log (3 c)}{-\log b}\right\}
$$

(See Figure 5b.)

Combining (c), (e) and (f) (note that these assumptions appear both in Examples 6.2 and 6.3 we get that the transversality condition holds. Namely, with the open set

$$
V:=(0,1)^{2} \times\left(\frac{\max \left(d_{2}, d_{3}\right)}{b-c}, \frac{\min \left(d_{1}, d_{3}\right)}{b-c}\right)
$$

the IFS $\widetilde{\mathcal{S}}$ satisfies the SSP. Hence by Lemma 1.2 the transversality condition holds. Hence, we can apply Theorem 4.1 for Example 6.2 in the same way we applied it for the Example 6.1. 
For the example 6.3 we are going to apply Theorem 4.2 here. The combination of (b) and Corollary 6.5 implies that $\nu_{x}$ has bounded density. Hence, Theorem 4.2 applies.

6.1.1. Phase transition in Example 6.3. Note that in Example 6.3 the parameter interval $I=\left(\frac{1}{\sqrt{3}}, 1\right)$ can be partitioned naturally into

$$
I_{1}:=\left(\frac{1}{\sqrt{3}}, \sqrt{\frac{2}{3}}\right] \text { and } I_{2}:=\left(\sqrt{\frac{2}{3}}, 1\right]
$$

Namely, the affinity dimension

$$
A(b, c):=1+\frac{\log (3 c)}{-\log b}
$$

is monotone increasing in both $b$ and $c$. So, for a fixed $c$ it takes its biggest value for $b=c / 2$. Clearly $c=\sqrt{2 / 3}$ is the solution of $A\left(\frac{c}{2}, c\right)=2$. That is for $c \in I_{1} \backslash E_{3}$ we have $\operatorname{dim}_{\mathrm{H}}(\Lambda)=A(b, c)$ for every $0<b \leq c / 2$. However, for $c \in I_{2} \backslash E_{3}$ it depends on the choice of $b$ if $\operatorname{dim}_{\mathrm{H}}(\Lambda)=A(b, c)$ or $\operatorname{dim}_{\mathrm{H}}(\Lambda)=2$. Now we fix a $c \in I_{2} \backslash E_{3}, u_{1}, u_{2}, u_{3}$ and $d_{1}, d_{2}, d_{3}$ satisfying the conditions of Example 6.3. We vary only $b$. So, the attractor will be denoted by $\Lambda_{b}$. To study the function

$$
b \mapsto \operatorname{dim}_{\mathrm{H}}\left(\Lambda_{b}\right), \quad b \in\left[0, \frac{c}{2}\right]
$$

first observe that for a $c \in I_{2}$ the function $A(\cdot, c)$ is monotone increasing and

$$
A\left(\frac{1}{3 c}, c\right)=2
$$

Then by Theorems 4.2 , for a $c \in I_{2} \backslash E_{3}$ we have:

$$
\operatorname{dim}_{\mathrm{H}} \Lambda_{b}= \begin{cases}1+\frac{\log (3 c)}{-\log b}, & \text { if } 0<b \leq \frac{1}{3 c} \\ 2, & \text { if } \frac{1}{3 c} \leq b \leq \frac{c}{2}\end{cases}
$$




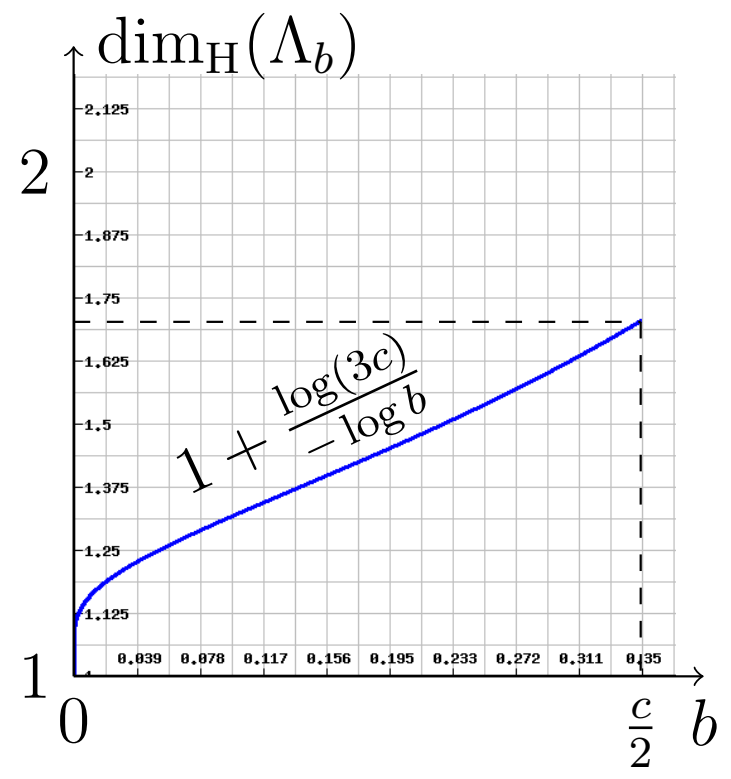

(A) $c=0.7<\sqrt{2 / 3}$.

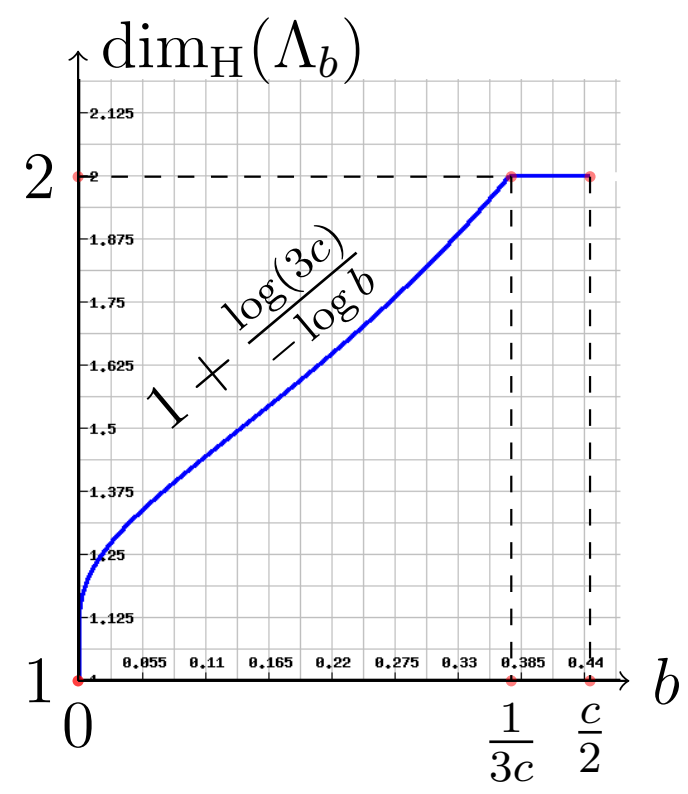

(B) $c=8 / 9>\sqrt{2 / 3}$.

Figure 6. The graphs of the continuous functions, with which the dimension coincides almost everywhere in Example 6.3. There is no phase transition if $c<\sqrt{2 / 3}$ and there is a phase transition at $b=c / 3$ for $\sqrt{2 / 3}<c<1$.

Which means that there is a phase transition at $b=c / 3$, where the graph of $b \mapsto$ $\operatorname{dim}_{\mathrm{H}} \Lambda_{b}$ is non-differentiable, although it is continuous on the interval $b \in\left(0, \frac{c}{2}\right]$.

6.1.2. The definition of exceptional parameter sets $E_{1}, E_{2}, E_{3}$. Let $E_{1}$ be the set of exceptional parameters

$$
E_{1}:=\left\{c \in\left(\frac{1}{2}, 1\right):(\mathrm{A} 3) \text { does not hold for the IFS }\{c x, c x+1\}\right\} .
$$

Let

$$
E_{2}:=\left\{c \in\left(\frac{1}{3}, 1\right): \text { (A3) does not hold for the IFS }\left\{c x+u_{1}, c x+u_{2}, c x+u_{3}\right\}\right\} .
$$

It follows from [22, Theorem A] that $E_{1}$ and $E_{2}$ are sets of Hausdorff dimension zero. About $E_{1}$ note that as long as $u_{1} \neq u_{2}$ the exceptional set is the same for all IFS $\left\{c x+u_{1}, c x+u_{2}\right\}$.

To define the third exceptional set first we need to state the following theorem [24, Theorem 2]

Theorem 6.4 (Simon, Tóth). For a natural number $N>1$ let $\mathfrak{m}_{\lambda}^{N}$ be the self-similar measure corresponding to the IFS $\{\lambda x+i\}_{i=0}^{N-1}$ and the uniform distribution of weights $\left\{\frac{1}{N}, \ldots, \frac{1}{N}\right\}$. Then there exists an exceptional set $R_{N} \subset\left(\frac{1}{N}, 1\right)$ having Lebesgue measure zero such that $\mathfrak{m}_{\lambda}^{N}$ is absolute continuous with $L^{2}$ density whenever $\lambda \in\left(\frac{1}{N}, 1\right) \backslash R_{N}$.

From this is immediate to see that the following Corollary holds: 
Corollary 6.5. Here we use the notation of Theorem 6.4. Let

$$
E_{3}:=\left\{c \in\left(\frac{1}{\sqrt{3}}, 1\right): c^{2} \in R_{3}\right\} .
$$

Then the Lebesgue measure of $E_{3}$ is zero and $\mathfrak{m}_{c}^{N}$ is absolutely continuous with continuous (consequently bounded) density for each $c \in\left(\frac{1}{\sqrt{3}}, 1\right) \backslash E_{3}$.

6.2. Example for the direction-y dominates case. Before we show an example for Theorem 5.1, we show a family of IFS of similarities, which satisfies Hochman's exponential separation condition.

Lemma 6.6. Let $\Psi:=\left\{x \mapsto \alpha x+\tau_{1}, x \mapsto \alpha x+\tau_{2}, x \mapsto \alpha x+\tau_{3}\right\}$ be an IFS on the real line such that $\alpha, \tau_{1}, \tau_{2} \in \mathbb{Q}$, and $\tau_{3} \notin \mathbb{Q}$. Then $\Psi$ satisfies Hochman's exponential separation condition.

The proof is similar to the proof of [13, Theorem 1.6].

Proof. Without loss of generality, we may assume that $\tau_{1}=0, \tau_{2}=1$, and $0<\tau_{3}<1$ irrational. For any finite length word $\bar{\imath} \in\{1,2,3\}^{*}$, let $\pi(\bar{\imath})=\sum_{k=0}^{|\bar{\imath}|} \tau_{i_{k}} \alpha^{k}$.

By (21), it is enough to show that there exists $\varepsilon>0$ and a sequence $n_{k}$ such that for any $\bar{\imath} \neq \bar{\jmath}$ with $|\bar{\imath}|=|\bar{\jmath}|=n_{k},|\pi(\bar{\imath})-\pi(\bar{\jmath})|>\varepsilon^{n_{k}}$. Suppose that this is not the case. That is, for every $\varepsilon>0$ there exists a $K$ such that for every $k \geq K$ there exist finite words $\bar{\imath}_{k}, \bar{\jmath}_{k}$ with $\left|\bar{\imath}_{k}\right|=\left|\bar{\jmath}_{k}\right|=k$ such that

$$
\left|\pi\left(\bar{\imath}_{k}\right)-\pi\left(\bar{\jmath}_{k}\right)\right|<\varepsilon^{k}
$$

By definition, $\alpha=p / q$, where $p, q \in \mathbb{N}$ relative primes and $q \neq 0$. Thus, for any $\bar{\imath} \neq \bar{\jmath}$ with $|\bar{\imath}|=|\bar{\jmath}|=k$,

$$
\pi(\bar{\imath})-\pi(\bar{\jmath})=\frac{p_{1}(\bar{\imath}, \bar{\jmath})}{q^{k}}+\tau_{3} \frac{p_{2}(\bar{\imath}, \bar{\jmath})}{q^{k}},
$$

where $p_{1}(\bar{l}, \bar{\jmath}), p_{2}(\bar{\imath}, \bar{\jmath}) \in \mathbb{N}$. Observe that

$$
\left|p_{i}(\bar{l}, \bar{\jmath})\right| \leq(2 q)^{k}
$$

for every $k \geq 1$ and $\bar{\imath}, \bar{\jmath}$ with $|\bar{\imath}|=|\bar{\jmath}|=k$. On the other hand, if $p_{2}(\bar{\imath}, \bar{\jmath})=0$ and $\bar{\imath} \neq \bar{\jmath}$ then $p_{1}(\bar{\imath}, \bar{\jmath}) \neq 0$, because of the rational root test of polynomials with integer coefficients. Thus, $|\pi(\bar{\imath})-\pi(\bar{\jmath})| \geq 1 / q^{k}$.

Hence, by taking $\varepsilon=1 /\left(16 q^{3}\right)$ and the sequence of words $\bar{\imath}_{k}, \bar{\jmath}_{k}$ with $\left|\bar{\imath}_{k}\right|=\left|\bar{\jmath}_{k}\right|=k$, given in the equation (107), we get

$$
\left|\frac{p_{1}\left(\bar{\imath}_{k}, \bar{\jmath}_{k}\right)}{p_{2}\left(\bar{\imath}_{k}, \bar{\jmath}_{k}\right)}-\tau_{3}\right|=\left|\pi\left(\bar{\imath}_{k}\right)-\pi\left(\bar{\jmath}_{k}\right)\right| \frac{q^{k}}{\left|p_{2}\left(\bar{\imath}_{k}, \bar{\jmath}_{k}\right)\right|} \leq \frac{1}{\left(16 q^{2}\right)^{k}}
$$

Therefore

$$
\left|\frac{p_{1}\left(\bar{\imath}_{k+1}, \bar{\jmath}_{k+1}\right)}{p_{2}\left(\bar{\imath}_{k+1}, \bar{\jmath}_{k+1}\right)}-\frac{p_{1}\left(\bar{\imath}_{k}, \bar{\jmath}_{k}\right)}{p_{2}\left(\bar{\imath}_{k}, \bar{\jmath}_{k}\right)}\right| \leq \frac{2}{\left(16 q^{2}\right)^{k}} .
$$

But since the left hand side is a rational number then

$$
\text { either }\left|\frac{p_{1}\left(\bar{\imath}_{k+1}, \bar{\jmath}_{k+1}\right)}{p_{2}\left(\bar{\imath}_{k+1}, \bar{\jmath}_{k+1}\right)}-\frac{p_{1}\left(\bar{\imath}_{k}, \bar{\jmath}_{k}\right)}{p_{2}\left(\bar{\imath}_{k}, \bar{\jmath}_{k}\right)}\right|>\frac{1}{(2 q)^{2 k+1}} \text { or }\left|\frac{p_{1}\left(\bar{\imath}_{k+1}, \bar{\jmath}_{k+1}\right)}{p_{2}\left(\bar{\imath}_{k+1}, \bar{\jmath}_{k+1}\right)}-\frac{p_{1}\left(\bar{\imath}_{k}, \bar{\jmath}_{k}\right)}{p_{2}\left(\bar{\imath}_{k}, \bar{\jmath}_{k}\right)}\right|=0 \text {. }
$$

Thus, for every $k$ large enough $p_{1}\left(\bar{\imath}_{k}, \bar{\jmath}_{k}\right) / p_{2}\left(\bar{\imath}_{k}, \bar{\jmath}_{k}\right) \equiv P / Q$, and by (108), $\tau_{3}=P / Q$, which contradicts to the assumption that $\tau_{3}$ is irrational.

Example 6.7. Let $\mathcal{S}$ be an IFS of the form (2), where

- $N=3$; 
- $c, b \in \mathbb{Q}$ such that $c<\min \{1 / 3, b\}, b<1$ and

$$
b<\min \left\{\sqrt{c}, c^{1+\frac{\log 3}{2 \log (3 c)}}\right\}
$$

- $d_{i} \bmod 3, d_{i+1} \bmod 3 \in \mathbb{Q}$ and $d_{i+2} \bmod 3 \notin \mathbb{Q}$ for an $i \in \mathbb{N}$;

- $u_{j \bmod 3}, u_{j+1} \bmod 3 \in \mathbb{Q}$ and $u_{j+2} \bmod 3 \notin \mathbb{Q}$ for an $j \in \mathbb{N}$.

(See Figure 7)

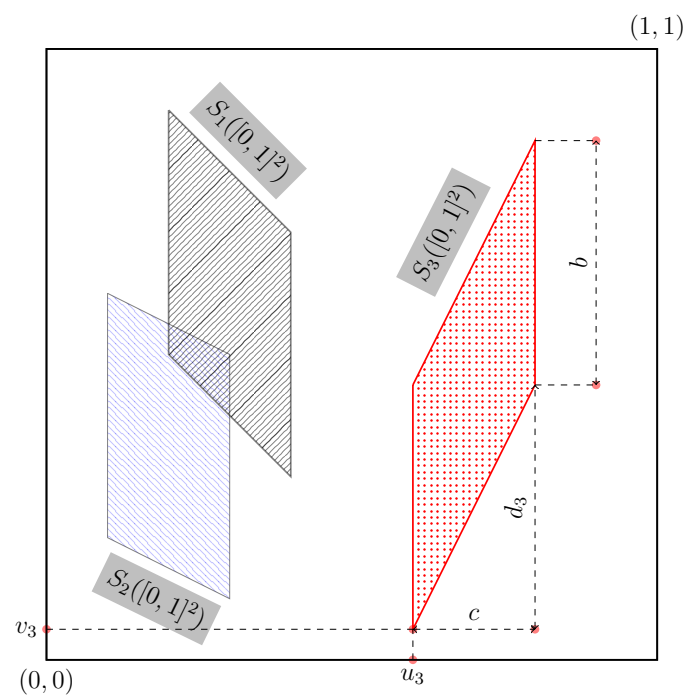

Figure 7. Figure for Example 6.7

Lemma 6.6 implies that $\mathcal{H}$ (defined in (4)) and the backward Furstenberg-Kifer IFS $\mathcal{B}$ (defined in (81)) satisfy Hochman's exponential separation condition. Moreover, the assumptions on $c, b$ verify that (C1), (C2) and (C5) hold in Assumption C.

\section{ApPENDiX}

Here we collect some consequences of the fact that a measure on the line is absolute continuous with $L^{q}$ density for a $q>1$.

Given a measure $\mu \ll \mathcal{L}_{1}$ supported by $[0,1]$ and we assume that there exists a $q>1$ such that the density $\varphi$ of $\mu$ satisfies:

$$
C_{q}:=\int_{0}^{1} \varphi(t)^{q} d t<\infty .
$$

Lemma 7.1. Let $\varphi(t)$ be the density of $\nu_{x}$. Assume that 109 holds. Then for any interval $I$

$$
\mu(I) \leq C_{q}^{1 / q}|I|^{1-1 / q} .
$$

Proof. By Jensen's inequality we have

$$
\int_{I} \varphi^{q}(t) d t \geq(\underbrace{\int_{I} \varphi(t) d t}_{\mu(I)})^{q} \cdot|I|^{1-q} .
$$

This completes the proof after some obvious algebraic manipulations. 
Definition 7.2. Given a real number $R>1$. We say that a not necessarily countable family $\mathcal{I}:=\left\{I_{i}\right\}_{i \in \Gamma}, I_{i} \subset[0,1]$ of closed intervals is an $R$-bad family if

$$
\frac{\mu\left(I_{i}\right)}{\left|I_{i}\right|}>R, \quad \forall i \in \Gamma
$$

We say that $\mathcal{I}$ is a disjoint $R$-bad family of intervals if $\mathcal{I}$ is an $R$-bad family and the intervals in $\mathcal{I}$ are pairwise disjoint.

Lemma 7.3. There exists a constant $c_{1}>0$ independent of everything such that for every $R>1$ if $\mathcal{I}$ is an $R$-bad family of intervals then

$$
\mu\left\{\bigcup_{i \in \Gamma} I_{i}\right\}<c_{1} \cdot R^{-(q-1)} .
$$

Proof for disjoint $R$-bad families. Fix an $R>1$. By Lemma 7.1 and equation (113) for every $i \in \Gamma$ we have

$$
C_{q} \geq \mu\left(I_{i}\right) \cdot\left(\frac{\mu\left(I_{i}\right)}{\left|I_{i}\right|}\right)^{q-1} .
$$

Using (111) and the fact that temporarily we assumed that $\mathcal{I}$ is a disjoint family we obtain that

$$
\mu\left(\bigcup_{i \in \Gamma} I_{i}\right) \leq C_{q} \cdot R^{-(q-1)}
$$

Proof for non-disjoint $R$-bad families. Fix an $R>1$. For every $i \in \Gamma$ and for every $x \in I_{i}$ let

$$
\widetilde{I}_{i}(x):=\left[x-\left|I_{i}\right|, x+\left|I_{i}\right|\right]
$$

Let $A:=\bigcup_{i} I_{i}$ and we form the family $\mathcal{B}$ of closed (one dimensional) balls $\left\{\widetilde{I}_{i}(x)\right\}_{i, x \in I_{i}}$. Then by definition we can apply Besicovitch's covering theorem (using the notation of [18, p. 30, Theorem 2.7 part (2)]) for $A$ and $\mathcal{B}$. From this theorem there is a constant $Q$ independent of everything such that we can find $Q$ sub-families $\mathcal{B}_{1}, \ldots, \mathcal{B}_{Q}$ of $\mathcal{B}$ such that

(a): for every $k, \mathcal{B}_{k}$ is a disjoint family. That is for any two distinct intervals $I, J \in$ $B_{k}$ we have $I \cap J=\emptyset$.

(b): If $J \in \mathcal{B}_{k}$ then $J=\left[x-\left|I_{i}\right|, x+\left|I_{i}\right|\right]$ for some $I_{i} \in \mathcal{I}$ and $x \in I_{i}$. Then

$$
\frac{\mu(J)}{|J|} \geq \frac{\mu\left(I_{i}\right)}{2\left|I_{i}\right|} \geq \frac{1}{2} \frac{\mu\left(I_{i}\right)}{\left|I_{i}\right|}>\frac{1}{2} \cdot R
$$

(c): $A \subset \bigcup_{k=1}^{Q} \bigcup_{J \in \mathcal{B}_{k}} J$

Using (a) and (b) for every $k, \mathcal{B}_{k}$ is a disjoint $R / 2$-bad family. Since we have already verified the assertion of the lemma for disjoint families we can apply (114) for $\mathcal{B}_{k}$ (with writing $R / 2$ instead of $R$ ) to get that for all $k=1, \ldots, Q$ :

$$
\mu\left\{\bigcup_{J \in \mathcal{B}_{k}} J\right\}<C_{q} \cdot 2^{q-1} \cdot R^{-(q-1)} .
$$


Using this and (c) above we obtain that

$$
\mu(A) \leq \underbrace{Q C_{q} 2^{q-1}}_{c_{1}} \cdot R^{-(q-1)} .
$$

From we get the following two corollaries:

Corollary 7.4. Let $\mathcal{I}_{n}^{\delta}=\left\{I_{i}^{n, \delta}\right\}$ be a family of intervals of $\left|I_{i}^{(n, \delta)}\right|=c^{n}$ and $\mu\left(I_{i}^{(n, \delta)}\right)>$ $c^{n(1-\delta)}$. Then $R=c^{-n \delta}$. So,

$$
\mu\left(\bigcup_{i} I_{i}^{(n, \delta)}\right)<c_{1} \cdot c^{n \delta(q-1)} .
$$

Corollary 7.5. Let $n \geq 1$ be arbitrary. Let $\omega \in\{1, \ldots, N\}^{n}$ and we write $I_{\boldsymbol{\omega}}$ for the interval which supports $\Pi_{x}(\boldsymbol{\omega})$ on the $x$-axis. We assume that $N c>1$. Let

$$
L_{n, \delta}:=\left\{t \in[0,1]: \sum_{|\boldsymbol{\omega}|=n} \mathbb{1}_{I_{\boldsymbol{\omega}}}(t)>(N c)^{n(1+\delta)}\right\}
$$

For every $t \in L_{n, \delta}$ let

$$
J_{t}:=\left[t-c^{n}, t+c^{n}\right] .
$$

Then

$$
\frac{\mu\left(J_{t}\right)}{\left|J_{t}\right|}>\frac{1}{2}(N c)^{n \delta}
$$

That is we may apply 112 for $\left\{J_{t}\right\}_{t \in L_{n, \delta}}$ to get that

$$
\mu\left(L_{n, \delta}\right) \leq \mu\left(\bigcup_{t \in L_{n, \delta}} J_{t}\right) \leq c_{1} 2^{q-1}(N c)^{-n \delta(q-1)} .
$$

recall that the constant $c_{1}$ was defined in (117), so it is independent of $\delta$.

Corollary 7.6. Assume $\nu_{x}$ has $L^{q}$ density for all $q>1$. Then for every $K>0$ and $q>1$ there exists $C=C(K, q)$ such that for every $\ell>0$ every interval $I$ of length $K c^{\ell}$ intersects at most $C(N c)^{\ell} c^{-\ell / q}$ intervals $h_{\boldsymbol{\tau}}([0,1]) ;|\boldsymbol{\tau}|=\ell$.

Proof. Denote by $Z$ the number of intervals $h_{\tau}([0,1]) ;|\tau|=\ell$ intersecting $I$. Each of those intervals is contained in the interval $I^{\prime}=B_{c^{\ell}}(I)$. Hence,

$$
\nu_{x}\left(I^{\prime}\right) \geq Z \cdot N^{-\ell} \text {. }
$$

Applying Lemma 7.1 to the interval $I^{\prime}$ we get the assertion.

\section{REFERENCES}

[1] B Bárány, Mark Pollicott, and K Simon. Stationary measures for projective transformations: the Blackwell and Furstenberg measures. Journal of Statistical Physics, 148(3):393-421, 2012.

[2] Balázs Bárány. Dimension of the generalized 4-corner set and its projections. Ergodic Theory and Dynamical Systems, 32(04):1190-1215, 2012.

[3] Balázs Bárány. On the Ledrappier-Young formula for self-affine measures. Math. Proc. Cambridge Philos. Soc., 159(3):405-432, 2015.

[4] Balázs Bárány and Antti Käenmäki. Ledrappier-Young formula and exact dimensionality of selfaffine measures. arXiv preprint arXiv:1511.05792, 2015.

[5] Balázs Bárány and Michał Rams. Dimension maximizing measures and local dimension spectrum for self-affine systems. to appear in Trans. Amer. Math. Soc., 2017. available at arXiv:1507.02829. 
[6] Balázs Bárány, Michał Rams, and Károly Simon. On the dimension of self-affine sets and measures with overlaps. Proceedings of the American Mathematical Society, 144(10):4427-4440, 2016.

[7] Kenneth Falconer and Tom Kempton. The dimension of projections of self-affine sets and measures. arXiv preprint arXiv:1511.03556, 2015.

[8] Kenneth Falconer and Jun Miao. Dimensions of self-affine fractals and multifractals generated by upper-triangular matrices. Fractals, 15(03):289-299, 2007.

[9] Kenneth J Falconer. The geometry of fractal sets, volume 85. Cambridge university press, 1986.

[10] Kenneth J Falconer. The Hausdorff dimension of self-affine fractals. Math. Proc. Camb. Phil. Soc, 103(3):339-350, 1988.

[11] De-Jun Feng and Huyi Hu. Dimension theory of iterated function systems. Communications on Pure and Applied Mathematics, 62(11):1435-1500, 2009.

[12] H. Furstenberg and Y. Kifer. Random matrix products and measures on projective spaces. Israel J. Math., 46(1-2):12-32, 1983.

[13] Michael Hochman. On self-similar sets with overlaps and inverse theorems for entropy. Ann. of Math. (2), 180(2):773-822, 2014.

[14] Michael Hochman and Boris Solomyak. On the dimension of Furstenberg measure for $s l \_2(r)$ random matrix products. arXiv preprint arXiv:1610.02641, 2016.

[15] Brian R. Hunt and Vadim Yu. Kaloshin. How projections affect the dimension spectrum of fractal measures. Nonlinearity, 10(5):1031-1046, 1997.

[16] Thomas Jordan, Mark Pollicott, and Károly Simon. Hausdorff dimension for randomly perturbed self affine attractors. Comm. Math. Phys., 270(2):519-544, 2007.

[17] Ka-Sing Lau. Self-similarity, $L^{p}$-spectrum and multifractal formalism. In Fractal geometry and stochastics (Finsterbergen, 1994), volume 37 of Progr. Probab., pages 55-90. Birkhäuser, Basel, 1995.

[18] Pertti Mattila. Geometry of sets and measures in Euclidean spaces: fractals and rectifiability. Number 44. Cambridge University Press, 1999.

[19] Yuval Peres and Wilhelm Schlag. Smoothness of projections, Bernoulli convolutions, and the dimension of exceptions. Duke Math. J., 102(2):193-251, 2000.

[20] Ariel Rapaport. On self-affine measures with equal Hausdorff and Lyapunov dimension. arXiv preprint arXiv:1511.06893, 2015.

[21] Pablo Shmerkin. On Furstenberg's intersection conjecture, self-similar measures, and the $l^{q}$ norms of convolutions. arXiv preprint arXiv:1609.07802, 2016.

[22] Pablo Shmerkin and Boris Solomyak. Absolute continuity of self-similar measures, their projections and convolutions. arXiv preprint arXiv:1406.0204, 2014.

[23] Károly Simon. The dimension theory of almost self-affine sets and measures. In Fractals, Wavelets, and their Applications, pages 103-127. Springer, 2014.

[24] Károly Simon and Hajnal R. Tóth. The absolute continuity of the distribution of random sums with digits $\{0,1, \ldots, m-1\}$. Real Anal. Exchange, 30(1):397-409, 2004/05.

[25] Boris Solomyak. On the random series $\sum \pm \lambda^{n}$ (an erdös problem). Annals of Mathematics, 142(3):611-625, 1995.

[26] Boris Solomyak. Measure and dimension for some fractal families. In Mathematical Proceedings of the Cambridge Philosophical Society, volume 124, pages 531-546. Citeseer, 1998.

[27] Peter Walters. An introduction to ergodic theory, volume 79 of Graduate Texts in Mathematics. Springer-Verlag, New York-Berlin, 1982.

(Balázs Bárány) Budapest University of Technology and Economics, MTA-BME Stochastics Research Group, P.O.Box 91, 1521 Budapest, Hungary

E-mail address: balubsheep@gmail.com

(Michał Rams) Institute of Mathematics, Polish Academy of Sciences, ul. Śniadeckich

8, 00-656 WARSZAWA, POLAND

E-mail address: rams@impan.pl

(Károly Simon) Budapest University of Technology and Economics, Department of Stochastics, Institute of Mathematics, 1521 Budapest, P.O.Box 91, Hungary

E-mail address: simonk@math.bme.hu 